\title{
EINIGE PROBLEME DER ENTWICKLUNG DER VERWALTUNGSGERICHTSBARKEIT IN DER REPUBLIK USBEKISTAN
}

\author{
I. Einleitung \\ II. Allgemeine Prinzipien des Rechtsschutzes der Bürger gegen Verwaltungshandeln \\ 1. Das Wesen der gerichtlichen Verwaltungskontrolle \\ 2. Grundstrukturen des Verwaltungsrechtsschutzes \\ III. Theoretische Grundlagen und einige Probleme der Entwicklung der \\ Verwaltungsgerichtsbarkeit \\ 1. Verwaltungsgerichtsbarkeit als ein wissenschaftliches Problem \\ a) Diskussion um die Schaffung einer Verwaltungsgerichtsbarkeit \\ b) Begriffe und verfassungsrechtliche Herausforderungen \\ c) Tragende Prinzipien eines rechtsstaatlichen Anforderungen entsprechenden \\ Verwaltungsprozesses \\ d) Verwaltungsgerichtsbarkeit als wichtige Einrichtung im öffentlichen Interesse \\ 2. Relevante gesellschaftliche, politische und rechtliche Bedingungen für die Akzeptanz der \\ Verwaltungsgerichtsbarkeit \\ a) Das Wesen der Verwaltungsreform als Systementscheidung \\ b) Rolle des Rechts in der rechtsstaatlichen Modernisierung des Staates \\ IV. Schlussbemerkungen
}


Dr. iur. Azamat

Egamberdiev,

Dozent der Taschkenter

Staatlichen Juristischen

Universität beim

Justizministerium der

Republik Usbekistan

orcid.org/0000-0001-5152-9885

\section{Einleitung}

Dem gerichtlichen Verwaltungsrechtsschutz kommt im Rechtsstaat eine grundlegende Bedeutung zu. Es ist jenes Institut, welches den Bürger aus der Rolle eines Objektes der Verwaltung befreit und in den Status eines Rechtssubjektes erhebt, das seine Ansprüche gegenüber den Trägern öffentlicher Verwaltung gleichberechtigt geltend machen, d.h. ein bestimmtes Handeln, Dulden oder Unterlassen fordern kann.

Im 2018 kam es in der Republik Usbekistan zu einem Ereignis, am 26. Januar d. J. wurde das Gesetzbuch der Republik Usbekistan über die Verwaltungsrechtspflege verabschiedet. Es ist erster fortschrittlicher Gesetzgebungsakt, der tradierte prozessuale Normen in ein neues öffentlichrechtliches Rechtsinstitut integriert, das die Rechte der Bürger schützt. Die Verabschiedung dieses Gesetzgebungsakts zeigt, dass Usbekistan, sich stützend auf seine eigene Rechtstradition, den Anschluss an die Entwicklungstendenzen des Verwaltungsverfahrens- und Verwaltungsprozessrechts der kontinental-europäischen Staaten sucht. In diesem Zusammenhang besteht aktuell die Notwendigkeit, Fragen der Verwaltungsgerichtsbarkeit, der bei der rechtsstaatlichen Modernisierung des Staates eine grundlegende Rolle zugewiesen wird, zu erforschen.

Vorliegender Aufsatz beschäftigt sich mit dem gegenwärtigen System gerichtlicher Verwaltungskontrolle in der Republik Usbekistan. Nach einem Überblick über die allgemeinen Prinzipien des Rechtsschutzes der Bürger gegen Verwaltungshandeln (II) werden kurze Aussagen zu einigen relevanten Problemen der öffentlichen Verwaltung und zur Entwicklung des Verwaltungsprozessrechts in Usbekistan (III) vorgestellt. Diese beziehen sich auf wichtige Aspekte wie das Wesen der Verwaltungsreform und des Rechtsstaates, die tragenden Prinzipien eines rechtsstaatlichen Anforderungen entsprechenden Verwaltungsprozesses, den Schutz des öffentlichen Interesses im Verwaltungsprozess, das Rechtsbewusstsein bzw. die Rechtskenntnisse der für Reformen relevanten Akteure u.a.

\section{Allgemeine Prinzipien des Rechtsschutzes der Bürger gegen Verwaltungshandeln \\ 1. Das Wesen der gerichtlichen Verwaltungskontrolle}

Die usbekische Verwaltungsrechtswissenschaft leitet das Recht auf die Verteidigung subjektiver Rechte und gesetzlicher Interessen der Bürger auf dem Gebiet der Verwaltung aus dem 
verwaltungsrechtlichen Status des Bürgers ab. Dieser bestimmt sich einerseits durch das System der durch verwaltungsrechtliche Normen bestimmten Rechte und Pflichten des Bürgers und andererseits durch die rechtlichen Garantien zur Realisierung dieser Rechte und Pflichten, einschließlich deren Schutz durch das Gesetz, dem Mechanismus ihres Schutzes durch die Organe der Staatsmacht sowie der Bereitstellung der Mittel, die der Bürger für den effektiven Schutz seiner Rechte benötigt ${ }^{1}$. Die Verteidigung subjektiver Rechte in Usbekistan wird auch als ein Aspekt der Sicherung der Gesetzlichkeit der öffentlichen Verwaltung verstanden. Als Mittel zur Sicherung der Gesetzlichkeit der Verwaltung und in diesem Sinne auch des Schutzes der Rechte der Bürger gelten vor allem die allgemeine staatliche Kontrolle, Verwaltungsaufsicht (umumiy ma'muriy nazorat), die staatsanwaltschaftliche Aufsicht (prokuror nazorati) und die gerichtliche Kontrolle (sud nazorati). Ebenso ist hierzu die gesellschaftliche Kontrolle (jamoatchilik nazorati) zu zählen.

Einen besonderen Mechanismus zum Schutz der Rechte der Bürger stellen jedoch Mittel dar, derer sich der Bürger unmittelbar selbst bedienen kann, vor allem durch Einlegung von Beschwerden und Klagen bei Gericht. So geht aus dem allgemeinen Beschwerde- und Antragsrecht (bei den Behörden) das allgemeine Recht auf Beschwerde bei Gericht hervor, das durch die Verfassung der Republik Usbekistan (Art. 44) garantiert wird und durch das bis 12.10.2018 geltende Gesetz „Über die Beschwerdeführung bei Gericht gegen Handlungen und Entscheidungen, welche die Rechte und Freiheiten der Bürger verletzen“" vom 30.08.1995² realisiert wurde ${ }^{3 .}$ Dabei wurden bis

${ }^{1}$ Egamberdiev,A.(2017). Current issues of transportinfrastructurein the Republic of Uzbekistan. $U P R$, special issue, pp. 443 f. [Egamberdiev A. Aktuelle Fragen der Verkehrsinfrastruktur in der Republik Usbekistan. UPR. 2017. Sonderheft. S. 443 f.].

${ }^{2}$ Gesetz der Republik Usbekistan „Über die Beschwerdeführung vor Gericht gegen Handlungen und Entscheidungen, die die Rechte und Freiheiten der Bürger verletzen“ (Fuqarolarning huquqlari va erkinliklari buzadigan xatti-haraqatlar va qarolar ustidan sudga shikoyat qilish to 'g'risida qonun) vom 30.08.1995 № O`RQ-108-I (außer Kraft getreten am 12.10.2018 aufgrund Gesetzes vom 11.10.2018 (№ O`RQ-496). URL: http://www.lex.uz/pages/getpage.aspx?lact_id=116757.

${ }^{3}$ Dabei entschied sich der Gesetzgeber gegen eine enumerative Auflistung von einzelnen Fallkategorien, in denen der Rechtsweg zum Gericht gegeben sein sollte, sondern für eine Generalklausel: Nach Art. 1 des Gesetzes vom 30.08.1995 war für Beschwerden gegen beliebige Handlungen (Entscheidungen) von Verwaltungsorganen und Amtspersonen der Rechtsweg $\mathrm{zu}$ den ordentlichen Gerichten eröffnet. Man muss aber anmerken, dass eine Reihe von verwaltungsrechtlichen Streitigkeiten noch in der Sowjetzeit für justitiabel erklärt wurde. So erlangte mit der Verfassung der Union der Sozialistischen Sowjetrepubliken (fortan - UdSSR) von 1977 bzw. der der Usbekischen Sozialistischen Sowjetrepublik von 1978 das Recht auf Beschwerde bei Gericht gegen Verwaltungshandeln Verfassungsrang. Die Verabschiedung des diese Verfassungsnorm der UdSSR ausführenden Gesetzes „Über die Beschwerdeführung bei Gericht gegenrechtswidrige Handlungen von Amtspersonen, die die Rechte der Bürger schmälern“ ließ jedoch bis zum 30.06.1987 auf sich warten. Mit ihm entschied man sich für die prinzipielle Möglichkeit, gegen jede beliebige Form von Verwaltungshandeln, das subjektive Rechte und gesetzlich geschützte Interessen der Bürger verletzt bzw. „schmälert“, Beschwerde bei Gericht einzulegen (Generalklausel), d.h. gegen die Auflistung konkreter Handlungen (Entscheidungen), die der Beschwerdeführung bei Gericht unterliegen.

Siehe dazu nur: Chamaneva, N.Yu. (1993). Appeal to a court of actions and decisions that violate the rights and freedoms of Russian citizens. State and law, no. 11, pp. 3 [Хаманева Н.Ю. Обжалование в суд действий и решений, нарушающих права и свободы граждан России. Государство и право. 1993. № 11. С. 3-12]. 
01.04.2018 die meisten öffentlich-rechtliche Streitigkeiten von den Zivilgerichten ${ }^{4}$ nach den zivilprozessrechtlichen Vorschriften entschieden, insbesondere nach Maßgabe der Kapitel 26-28 Zivilprozessordnung der Republik Usbekistan 5 .

Im Jahr 2016 hatte das Staatsoberhaupt die Notwendigkeit der alsbaldigen Verabschiedung des Gesetzbuches der Republik Usbekistan über die Verwaltungsrechtspflege, welches das Gerichtsverfahren in Verwaltungsstreitigkeiten regeln sollte, sowie auch die Schaffung von Verwaltungsgerichten bekräftigt ${ }^{6}$. Mit Wirkung zum 01.06.2017 wurde das Gerichtssystem Usbekistans um eine spezielle Verwaltungsgerichtsbarkeit ergänzt, die für öffentlich-rechtliche Streitigkeiten und

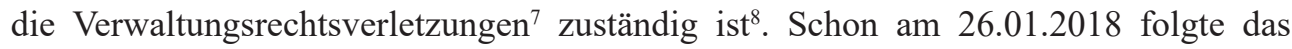
Gesetzbuch der Republik Usbekistan über die Verwaltungsrechtspflege, welches bereits am 01.04.2018 in Kraft trat ${ }^{9}$. Ein wichtiges Ergebnis der rechtsschöpferischen Tätigkeit im Bereich des Verwaltungsrechts ist darüber hinaus die Verabschiedung des Gesetzes der Republik Usbekistan „Über administrative Prozeduren“ am 08.01.2018 ${ }^{10}$.

${ }^{4}$ Solche Streitigkeiten wurden von den Zivilgerichten bis Ende Mai 2017 entschieden. Ab 01.06.2017 wurde das GerichtssystemUsbekistans um eine spezielle Verwaltungsgerichtsbarkeit ergänzt (siehe im weiteren Text).

${ }^{5}$ Die prozessualen Normen dieser Kapitel unterschieden sich in vielem von anderen Normen des Zivilprozessrechts und stellten ihrem Wesen nach Normen des Verwaltungsprozessrechts dar. Siehe die Zivilprozessordnung der Republik Usbekistan vom 30.08.1997 (außer Kraft getreten am 01.04.2018 aufgrund Gesetzes vom 29.01.2018 (№ O‘RQ-463). URL: http://lex.uz/docs/186098.

${ }^{6}$ So hat im Oktober 2016 der Präsident der Republik Usbekistan einen Erlass unterschrieben, in dem er das Oberste Gericht, das Oberste Wirtschaftsgericht, das Justizministerium und die Staatsanwaltschaft der Republik Usbekistan beauftragte, bereits im Jahr 2017 Vorschläge zur Schaffung von Verwaltungsgerichten und den Entwurf eines Gesetzbuches der Republik Usbekistan über die Verwaltungsrechtspflege auszuarbeiten. Siehe Erlass des Präsidenten Republik Usbekistan „Über die Maßnahmen zur weiteren Reformierung des Rechts- und Justizsystems, zur Verstärkung der Garantien eines zuverlässigen Schutzes der Rechte und Freiheiten der Bürger" vom 21.10.2016 № PF-4850. URL: http://www.lex.uz/pages/getpage.aspx?lact_id=3050491.

${ }^{7}$ Bei den Verwaltungsrechtsverletzungen handelt es sich um Verstöße gegen Verwaltungsvorschriften, die in Abgrenzung zu den Straftaten als Ordnungswidrigkeiten eingestuft werden und die sog. verwaltungsrechtliche Verantwortlichkeit (ma'muriy javobgarlik) begründen. Verwaltungsstraftatbestände enthalten der Besondere Teil des Gesetzbuchs der Republik Usbekistan über Verwaltungsverantwortlichkeit vom 22.09.1994 (in Kraft seit 01.04.1995, mit zahlreichen Änderungen), Gesetze (z.B. Zollgesetzbuch der Republik Usbekistan), normativrechtliche Akte von kompetenten Exekutivorganen (Amtspersonen).

${ }^{8}$ Im Februar 2017 wurde zudem ein Erlass des Präsidenten der Republik Usbekistan verabschiedet, der die Zusammenlegung des Obersten Gerichts und des Obersten Wirtschaftsgerichts der Republik Usbekistan anordnete und das Oberste Gericht der Republik Usbekistan zum einheitlichen höchsten Organ der Gerichtsgewalt in den Bereichen des Zivil-, Straf- Verwaltungs- und Wirschaftssrechts erklärte. Siehe Erlass des Präsidenten der Republik Usbekistan „Über Maßnahmen zur grundlegenden Vervollkommnung der Organisation und zur Effizienzverbesserung des Justizsystems der Republik Usbekistan“ vom 21.02.2017 № PF-4966. URL: http://www.lex.uz/pages/getpage.aspx?lact_id=3121087.

${ }^{9}$ Gesetzbuch der Republik Usbekistans über die Verwaltungsrechtspflege vom 26.01.2018 (in Kraft seit 01.04.2018, zuletzt geändert am 11.05.2019). URL: http://old.lex.uz/pages/getpage. aspx?lact_id=3527365. Siehe auch Beschluss № 17 des Plenums des Obersten Gerichts der Republik Usbekistan vom 19.05.2018. URL: http://www.lex.uz/ru/docs/3761216.

${ }^{10} \mathrm{Da}$ Verwaltungsverfahren und Verwaltungsprozess in funktionaler Hinsicht vielfach verwoben sind, wird die Hypothese aufgestellt, dass auch Verwaltungsverfahrens- und 
Das Zivilgesetzbuch der Republik Usbekistan ${ }^{11}$ sieht auch vor, dass der Schutz der bürgerlichen Rechte sowohl im verwaltungsbehördlichen Verfahren als auch vom Gericht, Wirtschaftsgericht und Schiedsgericht gewährt wird (Art. 10). Laut Artikel 12 des Zivilgesetzbuches wird der Akt eines Staatorgans oder der örtlichen Selbstverwaltung vom Gericht für unwirksam erklärt, wenn dieser Akt gegen die Gesetzgebung verstößt sowie auch die bürgerlichen Rechte und vom Gesetz geschützte Interessen eines Bürgers bzw. einer juristischen Person verletzt. Das heißt, dass für die Unwirksamerklärung eines Aktes von Staatsorganen oder Organen der örtlichen Selbstverwaltung zwei Bedingungen erfüllt sein müssen: erstens, der erlassene Akt entspricht nicht dem Gesetz bzw. einem anderen normativen Rechtsakt; zweitens, dieser Akt verletzt bürgerliche Rechte und vom Gesetz geschützte Interessen der natürlichen oder juristischen Person, die sich mit einem entsprechenden Antrag (Beschwerde) an das Gericht gewandt hat. Dabei kann über die Unwirksamkeit eines beliebigen Aktes der o.g. Organe ein Gericht ${ }^{12}$ oder Wirtschaftsgericht entscheiden.

Die gerichtliche Verwaltungskontrolle bezeichnet man in der Republik Usbekistan üblicherweise als „Gerichtsbarkeit in Verwaltungssachen“ (ma'muriy ishlar bo 'yicha odil sudlov) oder „Verwaltungsrechtspflege“ (ma'muriy sud ishlarini yuritish), seltener als „Verwaltungsjustiz“ (ma'muriy adliya) ${ }^{13}$. Während die Verwaltungsbehörde auf eine Beschwerde hin ein bestimmtes Verwaltungshandeln auf dessen Recht- und Zweckmäßigkeit überprüft, beschränkt sich das Gericht bei der Behandlung von Beschwerden und Klagen auf die Rechtmäßigkeitskontrolle.

Aktuelle Bedeutung bei der Reformierung des Verwaltungsverfahrensrechts und des Verwaltungsprozessrechts erlangt die Aufgabe der Verbesserung der Funktionsweise der Justiz als (von Legislative und Exekutive) unabhängiger Zweig der Staatsgewalt hinsichtlich der Gewährleistung der gerichtlichen Kontrolle über die Tätigkeit der

\footnotetext{
Verwaltungsprozessrecht auf normativer Ebene verknüpft sein müssen. In der Literatur besteht Einigkeit insoweit, als allgemein anerkannt wird, dass zwischen Verwaltungsverfahren und verwaltungsgerichtlichem Rechtsschutz ein Zusammenhang besteht. Es wird sowohl auf Parallelen und Berührungspunkte, auf Zusammenhänge und Wechselbeziehungen als auch auf weitgehende und grundlegende Unterschiede zwischen Verwaltungsverfahrensrecht und Verwaltungsprozessrecht hingewiesen. Siehe: Jochum, H. (2004). Verwaltungsverfahrensrecht und Verwaltungsprozessrecht: Die normative Konvexität von Verwaltungsverfahrens- und Verwaltungsprozessrecht und die Steuerungsleistung des materiellen Verwaltungsrechts (Vor diesem Hintergrund widmet sich Jochum der Frage nach dem normativen Verhältnis von Verwaltungsverfahrens- und Verwaltungsprozessrecht. Dabei werden rechtsdogmatische Verbindungslinien und Zusammenhänge zwischen verwaltungsrechtlichen, verwaltungsverfahrensrechtlichen und verwaltungsprozessrechtlichen Rechtsinstituten und Regelungskomplexen aufgedeckt, deren man sich vorher nicht bewusst war).

Bettermann, K.A. (1955). Administrative act and judge's decision. Memorial font for Walter Jellinek. Munich, pp. 361 ff. [Bettermann, K.A. (1955). Verwaltungsakt und Richterspruch. Gedächtnisschrift für Walter Jellinek. München, S. 361 ff.].

${ }^{11}$ Das Zivilgesetzbuch der Republik Usbekistan vom 25.12.1995 (in Kraft seit 01.03.1997, zuletzt geändert am 06.06.2019). URL: http://lex.uz/pages/getpage.aspx?lact_id=111181.

${ }^{12}$ Insbesondere: Verfassungsgericht, Verwaltungsgerichte, Zivilgerichte und Militärgerichte.

${ }^{13}$ Unter Rechtspflege versteht man traditionsgemäß die Anwendung von Rechtsnormen auf einen konkreten Streitfall (auf eine Tatsache, eine Handlung, eine Unterlassung oder ein Rechtsverhältnis) durch ein Gericht (einen Richter) in einem gesetzlich festgelegten Prozessverfahren mit verbindlicher Festlegung der Rechtsfolgen für die streitbeteiligten Personen.
} 
Verwaltung. Ein Richter soll Garant des Rechtsstaats und der Grundrechte der Menschen sein. Die Schaffung einer Verwaltungsgerichtsbarkeit neben dem Verfassungsgericht und den Wirtschaftsgerichten, Zivilgerichten und Militärgerichten als spezialisierter Zweig der Gerichtsbarkeit wirft auch die sehr wichtige Frage nach der Abgrenzung der sachlichen Zuständigkeit zwischen den betreffenden Gerichten ${ }^{14}$ auf. Man kann somit behaupten, dass mit der Einrichtung von Verwaltungsgerichten das usbekische Gerichtssystem zu seiner logischen Vollendung geführt wird. Eine aktive Anwendung des neuen Gesetzbuches durch die Gerichte wird die notwendigen Bedingungen schaffen, um die rechtliche Sicht auf das Wesen der Verwaltungsgerichtsbarkeit sowie auf das Öffentliche Recht im Ganzen zu erneuern. Im Großen und Ganzen kann man sagen, dass das Gesetzbuch der Republik Usbekistan über die Verwaltungsrechtspflege eine neue Plattform für die Entwicklung eines modernen Systems und der Organisation der Rechtsverhältnisse ist ${ }^{15}$.

\section{Grundstrukturen des Verwaltungsrechtsschutzes}

Das Verfahren der Verwaltungsgerichte in Rechtsstreitigkeiten, die aus verwaltungsrechtlichen Beziehungen resultieren, ist durch das Gesetzbuch der Republik Usbekistan über die Verwaltungsrechtspflege geregelt. Insbesondere ist das Verwaltungsgericht zuständig für Streitigkeiten betreffend den Schutz verletzter oder streitiger Rechte, Freiheiten und gesetzlichen Interessen der Bürger und juristischen Personen, die aus verwaltungsrechtlichen und anderen öffentlich-rechtlichen Rechtsbeziehungen resultieren, außer Streitigkeiten, die in die Zuständigkeit des Verfassungsgerichts der Republik Usbekistan, der Zivilgerichte, Wirtschaftsgerichte und Militärgerichte fallen (Art. 26). Rechtsstreitigkeiten über die Beschwerden von Militärangehörigen gegen Handlungen bzw. Entscheidungen von Armeeverwaltungsorganen und militärischen Amtspersonen werden von den Militärgerichten entschieden (Art. 31) ${ }^{16}$.

${ }^{14} \mathrm{Zu}$ den Fragen der Abgrenzung der sachlichen Zuständigkeit der Gerichte der ordentlichen Gerichtsbarkeit und des Verfassungsgerichts sowie auch der Wirtschaftsgerichte in Russland siehe beispielsweise:

Starilov, Yu.N. (1998). Administrative justice in Russia: problems of modern theory and development prospects. Eastern European Law, no. 3-4, pp. 217-252 [Старилов Ю.Н. Административная юстиция в России: проблемы современной теории и перспективы развития. Восточноевропейское право. 1998. № 3-4. С. 217-252].

Zhuykov, V.M. (1997). Judicial protection of the rights of citizens and legal entities. Moscow, pp. 195-301 [Жуйков В.М. Судебная защита прав граждан и юридических лиц. Москва, 1997. 320 c.].

${ }^{15}$ Der gegenwärtige Stand der theoretischen und praktischen Erarbeitung des Problems der Verwaltungsjustiz in den postsowjetischen Staaten gestattet es auch zu behaupten, dass eine spezialisierte Gerichtsbarkeit die Anerkennung und Einhaltung der Rechte und Freiheiten der Bürger gewährleistet und die Verantwortlichkeit der öffentlichen Gewalt für ihre Handlungen und Entscheidungen erhöht. Siehe beispielsweise:

Starilov, Yu.N. (1998). Administrative justice in Russia: problems of modern theory and development prospects. Eastern European Law, no. 3-4, pp. 217-252 [Старилов Ю.Н. Административная юстиция в России: проблемы современной теории и перспективы развития. Восточноевропейское право. 1998. № 3-4. С. 217-252].

Pudelka, J. (ed.) (2014). Administrative Process Code: conception, model law and commentary. Berlin: BWV [Verwaltungsprozesskodex: Konzeption, Modellgesetz und Kommentierung / J. Pudelka (Hrsg.). Berlin: BWV, 2014. 205 S.].

${ }^{16}$ Vgl. Art. 41 Abs. 3 GVG vom 14.12.2000 (zuletzt geändert am 11.05.2019). URL: http:// www.lex.uz/acts/68521. Man muss aber anmerken, dass vom Verwaltungsgericht Anträge bzw. 
Die in Art. 27 des Gesetzbuches über die Verwaltungsrechtspflege festgelegte Liste der sachlichen Zuständigkeiten der Verwaltungsgerichte umfasst folgende Verwaltungsstreitigkeiten:

- über die Anfechtung der normativen Rechtsakte der Behörden;

- über die Anfechtung der Handlungen (Untätigkeit) der Staatsverwaltung, anderer Organisationen, die mit einer verwaltungsrechtlichen Tätigkeit beauftragt wurden, der Selbstverwaltungsorgane und deren Amtspersonen;

- über die Anfechtung der Handlungen (Entscheidungen) der Wahlkommission;

- über die Anfechtung der Ablehnung der Vornahme rechtsgeschäftlicher Handlungen des Notars oder des Standesamts bzw. Handlungen (Untätigkeit) des Notars oder Standesamts;

- über die Anfechtung der Verweigerung der staatlichen Registrierung oder ihre Nichtvornahme innerhalb der festgelegten Frist.

Diese Liste von Verwaltungsstreitigkeiten ist jedoch nicht abschließend. So wird in Art. 27 Abs. 2 des Gesetzbuches über die Verwaltungsrechtspflege festgelegt, dass das Verwaltungsgericht auch andere Streitigkeiten betreffend den Schutz der verletzten oder streitigen Rechten, Freiheiten und gesetzlichen Interessen der Bürgern und juristischen Personen, die aus verwaltungsrechtlichen sowie anderen öffentlich-rechtlichen Rechtsbeziehungen resultieren und der Zuständigkeit des Verwaltungsgerichts zugewiesen sind, entscheiden kann. Falls verschiedene miteinander verbundene Begehren vorgebracht werden, wobei einige Begehren der Zuständigkeit der Verwaltungsgerichte und andere der Zuständigkeit der Zivilgerichte zugewiesen sind, so soll über alle Begehren das Zivilgericht entscheiden (Art. 26).

Gemäß Art. 37 Abs. 3 GVG entscheiden die Wirtschaftsgerichte diejenigen Streitigkeiten, die in Zivilrechtsverhältnissen zwischen Wirtschaftssubjekten entstanden sind, Streitigkeiten zwischen juristischen Personen sowie auch über Verwaltungsrechtsverletzungen, die der Zuständigkeit des Wirtschaftsgerichts zugewiesen sind. Jedoch bestimmt die am 09.01.2018 neu verabschiedete Wirtschaftsprozessordnung der Republik Usbekistan (fortan - WPO), dass die Wirtschaftsgerichte auch über Rechtsstreitigkeiten entscheiden, die aus verwaltungsrechtlichen Beziehungen resultieren ${ }^{17}$. Insbesondere folgende verwaltungsrechtliche Rechtsstreitigkeiten sind der Zuständigkeit des Wirtschaftsgerichts zugewiesen:

Beschwerden, nicht Klagen geprüft werden. Damit folgt der Gesetzgeber der oben erwähnten Tradition des sowjetischen Beschwerderechts, wonach Vorschläge (takliflar), Anträge (arizalar) und Beschwerden (shikoyatlar) als Formen von Bürgerappellen (fuqarolarning murojaatlari) gelten, wobei die Beschwerden schrittweise auch in die Prüfungskompetenz der Gerichte übertragen wurden. Hier hat der Gesetzgeber die Chance verpasst, den Bürger aus der Position eines Antragsoder Bittstellers (arizachi/shikoyatchi) in die eines Klägers (da'vogar), der dem Verwaltungsorgan im Gerichtsverfahren gleichberechtigt gegenübertritt, zu erheben. $\mathrm{Zu}$ dieser Problematik siehe auch: Hanisch, S. (1996). Der gerichtliche Rechtsschutz gegen Verwaltungshandeln in der Russischen Föderation: Allgemeine Darstellung von Theorie und Rechtslage unter Einbeziehung aktueller Gerichtsentscheidungen (Langfassung des Seminarreferats vom 08.02.1996 am Osteuropa-Institut der Freien Universität Berlin, unveröffentlicht), S. 12-13.

${ }^{17}$ Hauptkriterien, nach denen die Zuständigkeit der Verwaltungsgerichte von der Zuständigkeit der Wirtschaftsgerichte abzugrenzen ist, sind die Verknüpftheit einer verwaltungsrechtlichen Streitigkeit mit wirtschaftlichen Beziehungen oder Beziehungen aus unternehmerischer Tätigkeit sowie auch die subjektive Zusammensetzung der Parteien (natürlichen oder juristische Personen). 
- Klagen auf die Aussetzung der Vollziehbarkeit eines Vollstreckungstitels oder eines anderen Aktes, auf dessen Grundlage im unstreitigen Verfahren (akseptsiz) eine Geldforderung beige trieben wird, nach Art. 26 Abs. 1 Ziff. 12 Wirtschaftsprozessordnung (WPO);

- Klagen gegen die Einziehung von Geldstrafen von juristischen Personen und Bürgern durch Organe, die Kontrollfunktionen wahrnehmen, wenn nicht durch Gesetz ein unstreitiges Verfahren ihrer Einziehung vorgesehen ist, nach Art. 26 Abs. 1 Ziff. 13 WPO;

- Klagen auf die Rückerstattung von Geldmitteln, die von Organen, die Kontrollfunktionen wahrnehmen, im unstreitigen Verfahren (akseptsiz) unter Verletzung der Anforderungen der Gesetzgebung beigetrieben wurden, nach Art. 26 Abs. 1 Ziff. 14 WPO.

\section{Theoretische Grundlagen und einige Probleme der Entwicklung der Verwaltungsgerichtsbarkeit}

\section{Verwaltungsgerichtsbarkeit als ein wissenschaftliches Problem}

Der Verwaltungsgerichtsbarkeit als ein wissenschaftliches Problem sind weltweit unzählige Arbeiten gewidmet worden, in denen diese unter historischen, juristischinhaltlichen und rechtsvergleichenden Aspekten betrachtet wird. Ein Blick auf die Rechtsund Verwaltungswissenschaft in Zentralasien offenbart ein gewisses Widerstreben, sich mit neuen Ansätzen überhaupt zu befassen ${ }^{18}$. Neuerem usbekischem Schrifttum zu rechtsdogmatischen Fragen des Verwaltungsrechtsschutzes in Usbekistan mangelt es an Tiefe und Substanz ${ }^{19}$.

\footnotetext{
${ }^{18}$ Pudelka, J., Deppe, J. (2017). General administrative law in development in Central Asia. Ost/Mag, pp. 3. URL: https://www.ostinstitut.de/documents/publikationen/Pudelka Deppe_Allgemeines_Verwaltungsrecht_in_Zentralasien_in_der_Entwicklung_OL_2 2017.pdf [Pudelk̄a J., Deppe J. Allgemeines Verwaltungsrecht in Z̄entralasien in der Entwick̄lüng. 2017. Ost/Mag. URL: https://www.ostinstitut.de/documents/publikationen/Pudelka_Deppe Allgemeines_Verwaltungsrecht_in_Zentralasien_in_der_Entwicklung_OL_2_2017.pdf].

${ }^{19}$ In letzten Jahren wurden nur einige wenige Artikel veröffentlicht, deren Autoren die rechtlichen Besonderheiten dieses Rechtsinstituts und seine Entwicklungsperspektiven in Usbekistan zu erforschen suchten.

Siehe zum Beispiel: Chvan, L.B. (2014). Administrative justice and Central Asian countries: Quo Vadis. Public Law yearbook 2014. Administrative law: comparative legal approaches. Moscow: Infotropic Media, pp. 201-221 [Хван Л.Б. Административная юстиция и страны Центральной Азии: Quo Vadis. Ежегодник публичного права 2014. Административное право: сравнительно-правовые подходы. Москва : Инфотропик Медиа, 2014. С. 201-221].

Nematov, Zh.N. (2014). Administrative justice in the Republic of Uzbekistan, in the Russian Federation and Japan: comparative legal analysis. The Constitution of the Russian Federation and its influence on the practice of state building and the development of the country's legal system: materials of the International Scientific and Practical Conference dedicated to the 55th anniversary of the Law Faculty of Voronezh State University (Voronezh, October 17-18, 2013). Voronezh, pp. 189-201 [Нематов Ж.Н. Административная юстиция в Республике Узбекистан, в Российской Федерации и Японии: сравнительно-правовой анализ. Конституция Российской Федерации и ее влияние на практику государственного строительства и развитие правовой системь cтраны : материалы Международной научно-практической конференции, посвященной 55-летнему юбилею юридического факультета Воронежского государственного университета, г. Воронеж, 17-18 октября 2013 г. Воронеж : Изд. дом ВГУ, 2014. С. 189-201].
} 
Die vorliegenden Forschungen in Usbekistan folgen zudem der sowjetischen und russischen Verwaltungsrechtslehre ${ }^{20}$.

Chvan, L.B. (2015). Legal innovations in administrative justice of the countries of the European Union: possibilities of application in the countries of Central Asia. Public law yearbook 2015. Administrative process. Moscow: Infotropic Media, pp. 95-113 [Хван Л.Б. Правовые новации в административном правосудии стран Европейского Союза: возможности применения в странах Центральной Азии. Ежегодник публичного права 2015. Административный прочесс. Москва : Инфотропик Медиа, 2015. С. 95-113].

Musaev, A. (2015). The legislation on administrative proceedings in the Republic of Uzbekistan. Public law yearbook 2015. Administrative process. Moscow: Infotropic Media, pp. 172-179 [Мусаев А. Законодательство об административном судопроизводстве в Республике Узбекистан. Ежегодник публичного права 2015. Административный процесс. Москва : Инфотропик Медиа, 2015. С. 172-179].

Egamberdiev, A. (2016). Administrative justice as an integral element of a democratic state of law. Fragen der Issues of further improving the legal framework for the organization of law enforcement in the Republic of Uzbekistan: materials of a scientific and practical conference. Tashkent, pp. 85 f. [Эгамбердиев А. Административная юстиция как неотъемлемый элемент демократического правового государства. Вопросы дальнейшего совершенствования правовых основ организачии правоохранительной деятельности в Республике Узбекистан : материалы научно-практической конференции. Ташкент, 2016. С. 85-90].

${ }^{20}$ Rechtswissenschaftler untersuchten das Problem der Verwaltungsjustiz auch im zaristischen Russland, desgleichen in der Sowjetzeit und in der Russischen Föderation ab 1991 bis zur Gegenwart. Siehe beispielsweise:

Petuchov, G.E. (1974). Administrative Justice in Tsarist Russia. Jurisprudence, no. 5, pp. 72-80 [Петухов Г.Е. Административная юстиция в царской России. Правоведение. 1974. № 5. C. 72-80].

Korf, S.A. (1910). Administrative Justice in Russia: in 2 vol. Saint Petersburg [Корф С.А. Административная юстиция в России : в 2 т. Санкт-Петербург, 1910. 528+507 с.].

Zagryatskov, M.D. (1925). Administrative justice and the right to complain in theory and law. Moscow [Загряцков М.Д. Административная юстиция и право жалобы в теории и законодательстве. Москва, 1925. 244 с.].

Boytsova, V.V. (1993). Do we need administrative justice? Soviet justice, no. 7, pp. $12 \mathrm{f}$ [Бойцова В.В. Нужна ли нам административная юстиция? Советская юстищия. 1993. № 7. C. $12-15]$.

Boytsova, V.V., Boytsov, V.Ya. (1994). Administrative justice: to continue the discussion on the content and meaning. State and law, no. 5, pp. 42-53 [Бойцова В.В., Бойцов В.Я. Административная юстиция: к продолжению дискуссии о содержании и значении. Государство и право. 1994. № 5. С. 42-53].

Salishcheva, N.G. (1994). On some methods of protecting and protecting the rights, freedoms and legitimate interests of citizens in the field of executive power in the Russian Federation. Lukasheva, E.A. (ed.). The Constitution of the Russian Federation and the improvement of mechanisms for protecting human rights. Moscow, pp. 78-93 [Салищева Н.Г. О некоторых способах защиты и охраны прав, свобод и законных интересов граждан в сфере деятельности исполнительной власти в Российской Федерации. Конституции Российской Федерации и совершенствование механизмов защиты прав человека / отв. ред. Е.А. Лукашева. Москва, 1994. C. 78-93].

Skitovich, V.V. (1995). Justice in cases arising from administrative-legal relations: historical experience and prospects. State and law, no. 8, pp. 22-29 [Скитович В.В. Правосудие по делам, возникающим из административно-правовых отношений: исторический опыт и перспективы. Государство и право. 1995. № 8. С. 22-29].

Starilov, Yu.N. (1995). Russian officials on the path to professionalism. Russian justice, no. 9, pp. 40-42 [Старилов Ю.Н. Чиновники России на пути к профессионализму. Российская юстищия. 1995. № 9. С. 40-42]. 


\section{a) Diskussion um die Schaffung einer Verwaltungsgerichtsbarkeit}

Das Institut der Verwaltungsgerichtsbarkeit, das in verschiedenen westlichen Ländern (materiell) entweder über die ordentlichen Gerichte oder durch spezielle Verwaltungsgerichte realisiert wird, kann man als ,, besonderes prozessuales Verfahren der Entscheidung verwaltungsrechtlicher Streitigkeiten zwischen dem Bürger und staatlichen Verwaltungsorganen und Amtspersonen "definieren. In der Sowjetunion waren Verwaltungsstreitigkeiten lange Zeit nicht justitiabel, und bis zum Ende der Sowjetunion wurde keine allgemeine Rechtswegeröffnung in verwaltungsrechtlichen Streitigkeiten (Generalklausel) geschaffen. Ebenso gab es kein eigenständiges Verwaltungsprozessrecht. Verwaltungsrechtliche Streitigkeiten wurden - wie in den meisten anderen sozialistischen Ländern auch - innerhalb der ordentlichen Gerichtsbarkeit nach den jeweiligen Zivilprozessgesetzen behandelt, die teilweise Sonderregelungen für öffentlich-rechtliche Streitigkeiten vorsahen ${ }^{21}$.

In der russischen Literatur der 1990er Jahre wurden die Chancen für die Schaffung spezieller Verwaltungsgerichte eher negativ beurteilt, obwohl nicht ausgeschlossen und vielfach die Hoffnung geäußert wird, dass sich das Institut der Verwaltungsgerichtsbarkeit in den postsowjetischen Staaten durchsetzt ${ }^{22}$. Dies wird hauptsächlich mit praktischen, weniger mit theoretischen Erwägungen begründet. So seien die Bürger an die ordentlichen Gerichte als bevölkerungsnäheste Institutionen gewöhnt und eine Erweiterung des Gerichtsapparats sei nicht wünschenswert.

Studenikina, M.S. (1997). Administrative justice needs clear legal regulation. Journal of Russian Law, no. 6, pp. 11-20 [Студеникина М.С. Административная юстиция нуждается в четком правовом регулировании. Журнал российского права. 1997. № 6. С. 11-20].

Timoshenko, J.G. (1997). UK Administrative justice. Journal of Russian Law, no. 5, pp. 128-136 [Тимошенко И.Г. Административная юстиция в Великобритании. Журнал российского права. 1997. № 5. С. 128-136].

Lafitskiy, V.I. (1997). US Administrative justice. Journal of Russian Law, no. 7, pp. 117-125 [Лафитский В.И. Административная юстиция в США. Журнал российского права. 1997. № 7. С. 117-125].

Kostareva, T.A. (1997). About the judicial system of Germany. Journal of Russian Law, no. 8, pp. 128-138 [Костарева Т.А. О судебной системе ФРГ. Журнал российского права. 1997. № 8. C. 128-138].

${ }^{21}$ Starilov, Yu.N. (1995). Russian officials on the path to professionalism. Russian justice, no. 9, pp. 40-42 [Старилов Ю.Н. Чиновники России на пути к профессионализму. Российская юстищия. 1995. № 9. С. 40-42].

${ }^{22}$ Boytsova, V.V. (1993). Do we need administrative justice? Soviet justice, no. 7, pp. 13 [Бойцова В.В. Нужна ли нам административная юстиция? Советская юстищия. 1993. № 7. C. 12-15].

Boytsova, V.V., Boytsov, V.Ya. (1994). Administrative justice: to continue the discussion on the content and meaning. State and law, no. 5, pp. 51-53 [Бойцова В.В., Бойцов В.Я. Административная юстиция: к продолжению дискуссии о содержании и значении. Государство и право. 1994. № 5. С. 42-53].

Skitovich, V.V. (1995). Justice in cases arising from administrative-legal relations: historical experience and prospects. State and law, no. 8, pp. 27-29 [Скитович В.В. Правосудие по делам, возникающим из административно-правовых отношений: исторический опыт и перспективы. Государство и право. 1995. № 8. С. 22-29].

Starilov, Yu.N. (1995). Russian officials on the path to professionalism. Russian justice, no. 9, pp. 42 [Старилов Ю.Н. Чиновники России на пути к профессионализму. Российская юстищиия. 1995. № 9. С. 40-42]. 
Die Prüfung von Bürgerbeschwerden durch die Volksgerichte (jetzt Amtsgerichte) habe sich zu einer Tradition verfestigt ${ }^{23}$. Außerdem stelle das Zivilprozessverfahren in seiner Universalität eine durchaus annehmbare Prozedur auch für die Prüfung verwaltungsrechtlicher Fälle dar ${ }^{24}$. Dabei wurde jedoch nicht ausgeschlossen, dass die Schaffung spezieller Spruchkörper nicht nur in Russland, sondern auch in anderen GUSLändern notwendig sei. Vorerst gelte es, sich auf die Vervollkommnung der bestehenden gerichtlichen Mechanismen, auf die genaue Abgrenzung der verschiedenen sachlichen Zuständigkeiten zu konzentrieren. Es müssen verschiedene Rechtswege erhalten bleiben. Je mehr Rechtswege bestehen, desto demokratischer sei das Gerichtssystem ${ }^{25}$.

Die russischen Rechtswissenschaftler, welche die Probleme der Verwaltungsgerichtsbarkeit untersucht haben, verweisen auf deren unterschiedliche Eigenschaften ${ }^{26}$. Im Wesentlichen hebt man drei allgemeine Merkmale hervor: 1) ihre Zuständigkeit für Streitigkeiten, die auf dem Gebiet der Verwaltung zwischen Bürgern oder juristischen Personen einerseits und Verwaltungsorganen andererseits entstehen (also Streitsachen über das Verwaltungsrecht); 2) die Schaffung eines speziellen Organs für die Verhandlung der genannten Streitigkeiten; 3) die Verhandlung der Streitigkeiten unter Beachtung einer gerichtsprozessualen Form ${ }^{27}$.

Für die Republik Usbekistan, die die Prozesse der rechtsstaatlichen Modernisierung der Staates verhältnismäßig unlängst begann und nunmehr schon ein eigenes Verwaltungsverfahrens- und Verwaltungsprozessrecht hat, ist sehr wichtig, eine eigene wissenschaftlich begründete Konzeption des Aufbaus und der Ausübung einer nationalen Verwaltungsgerichtsbarkeit zu schaffen. In der Literatur wird die Meinung

${ }^{23}$ Boytsova, V.V., Boytsov, V.Ya. (1994). Administrative justice: to continue the discussion on the content and meaning. State and law, no. 5, pp. 45 [Бойцова В.В., Бойцов В.Я. Административная юстиция: к продолжению дискуссии о содержании и значении. Государство и право. 1994. № 5. С. 42-53].

${ }^{24}$ Chamaneva, N.Yu. (1993). Appeal to a court of actions and decisions that violate the rights and freedoms of Russian citizens. State and law, no. 11, pp. 3 [Хаманева Н.Ю. Обжалование в суд действий и решений, нарушающих права и свободы граждан России. Государство и право. 1993. № 11. С. 3-12].

Skitovich, V.V. (1995). Justice in cases arising from administrative-legal relations: historical experience and prospects. State and law, no. 8, pp. 27 f [Скитович В.В. Правосудие по делам, возникающим из административно-правовых отношений: исторический опыт и перспективы. Государство и право. 1995. № 8. С. 22-29].

${ }^{25}$ Hanisch, S. (1996). Der gerichtliche Rechtsschutz gegen Verwaltungshandeln in der Russischen Föderation: Allgemeine Darstellung von Theorie und Rechtslage unter Einbeziehung aktueller Gerichtsentscheidungen (Langfassung des Seminarreferats vom 08.02.1996 am Osteuropa-Institut der Freien Universität Berlin, unveröffentlicht), S. 12-13.

${ }^{26}$ Starilov, Yu.N. (1998). Administrative justice in Russia: problems of modern theory and development prospects. Eastern European Law, no. 3-4, pp. 217-252 [Старилов Ю.Н. Административная юстиция в России: проблемы современной теории и перспективы развития. Восточноевропейское право. 1998. № 3-4. С. 217-252].

Chechot, D.M. (1973). Administrative justice (theoretical problems). Leningrad, pp. 29-31 [Чечот Д.М. Административная юстиция (теоретические проблемы). Ленинград, 1973. 134 c.].

${ }^{27}$ Chechot, D.M. (1973). Administrative justice (theoretical problems). Leningrad, pp. 29-31 [Чечот Д.М. Административная юстиция (теоретические проблемы). Ленинград, 1973. 134 c.]. 
vertreten, dass angesichts der in den GUS-Ländern bestehenden Voraussetzungen der ,, deutsche Typ “ der Verwaltungsjustiz der passendste sei ${ }^{28}$. Im deutschsprachigen Schrifttum wird Verwaltungsgerichtsbarkeit verstanden als über die Verwaltung ausgeübte Gerichtsbarkeit, historisch entstanden als Instrument zur Sicherung des Rechtsstaates, d.h. zur Gewährleistung der Normengebundenheit staatlicher Tätigkeit und der Freiheitsrechte des Einzelnen ${ }^{29}$. Dieser Entstehungszusammenhang legt es nahe, die Herausbildung eines durch die genannten Komponenten geprägten Gemeinwesens unter staatstheoretischem und staatshistorischem Aspekt im Laufe der Geschichte zurückzuverfolgen.

Ausgehend von den Ergebnissen einer vorläufigen Analyse der vorherrschenden doktrinellen Ansätze deutscher Rechtswissenschaftler in diesem Bereich wie auch der etablierten Praxis der Rechtsanwendung in Deutschland sowie der Verwandtschaft der Rechtssysteme Deutschlands und Usbekistans (römisch-germanischer Rechtskreis) ist für das Rechtssystem Usbekistans gerade die zu untersuchende deutsche Erfahrung besonders wertvoll und nützlich. Die deutsche Erfahrung in diesem Bereich ist besonders relevant, weil Deutschland tief verwurzelte demokratische Traditionen aufweist und viele Verfassungsprinzipien, hinsichtlich derer in Usbekistan (sowie in anderen ehemaligen Sowjetrepubliken) teilweise erhebliche Umsetzungsdefizite bestehen (im vorliegenden Kontext insbesondere Gewaltenteilung und Rechtsstaatlichkeit), in Deutschland Rechtswirklichkeit sind. In diesem Zusammenhang kann ein Rechtsvergleich zwischen Verwaltungsrechtssystemen dazu dienen, die Möglichkeit einer transnationalen Verwaltungszusammenarbeit zu sondieren, um gegebenenfalls Änderungsbedarf festzustellen ${ }^{30}$. Darüber hinaus könnte eine umfassende Erforschung der deutschen Erfahrung in diesem Bereich im Ganzen helfen, die rechtswissenschaftliche Diskussion in Usbekistan zu bereichern, um wichtige Impulse für entsprechende Gesetzgebungsvorhaben in Usbekistan zu vermittel ${ }^{31}$.

${ }^{28}$ Vedernikova, O.N. (1997). Administrative justice (experience of foreign countries). Judicial reform: results, priorities, prospects: conference proceedings. Series "Scientific Papers". Moscow, no. 47, pp. 60 [Ведерникова О.Н. Административная юстиция (опыт зарубежных стран). Судебная реформа: итоги, приоритеть, перспективы : материалы конференции. Серия «Научные доклады». Москва, 1997. № 47. С. 52-60].

Starilov, Yu.N. (1998). Administrative justice in Russia: problems of modern theory and development prospects. Eastern European Law, no. 3-4, pp. 217-252 [Старилов Ю.Н. Административная юстиция в России: проблемы современной теории и перспективы развития. Восточноевропейское право. 1998. № 3-4. С. 217-252].

Pudelka, J. (ed.) (2014). Administrative Process Code: conception, model law and commentary. Berlin: BWV [Verwaltungsprozesskodex: Konzeption, Modellgesetz und Kommentierung / J. Pudelka (Hrsg.). Berlin: BWV, 2014. 205 S.]

${ }^{29}$ Sodan, H., Ziekow, J. (2018). Basic course in public law, constitutional and administrative law. 8th ed. Berlin, pp. 737, Rndr. 3 [Sodan H., Ziekow J. Grundkurs Öffentliches Recht, Staatsund Verwaltungsrecht. 8. Aufl. Berlin, 2018. S. 737, Rndr. 3].

${ }^{30}$ Sommermann, K.-P. (2013). Knowledge interests of comparative law in administrative law. Gamper, A., Verschraegen, B. (eds.). Comparative law as a legal method of interpretation. IGKK Series, Vol. IV. Wien, pp. 199 [Sommermann K.-P. Erkenntnisinteressen der Rechtsvergleichung im Verwaltungsrecht. Rechtsvergleichung als juristische Auslegungsmethode / A. Gamper, B. Verschraegen (Hrsg.). IGKK Series, Bd. IV. Wien, 2013. S. 199].

${ }^{31}$ Nach der Konzeption zur Einführung eines Verwaltungsprozesskodex, die durch eine von der GIZ geleitete Arbeitsgruppe erarbeitet wurde, sollten die Verwaltungsgerichte grundsätzlich 


\section{b) Begriffe und verfassungsrechtliche Herausforderungen}

Eines der wichtigsten Probleme der gegenwärtigen Entwicklung des Verwaltungsprozessrechts Usbekistans ist das Fehlen eines einheitlichen Ansatzes zur Definition der Begriffe in diesen Rechtsbereich. Insbesondere wurden einheitliche und unter Wissenschaftlern wie Praktikern gleichermaßen anerkannte Definitionen folgender Begriffe bisher nicht formuliert: „Verwaltungsprozess“ (ma'muriy prosess), „Verwaltungsprozessrecht" (ma'muriy-prosessual huquq), „Verwaltungsrechtspflege“ (ma'muriy sud ishlarni yuritish), „Verwaltungsakt" (ma'muriy hujjat) ${ }^{32}$. Diese Begriffe sind nach wie vor in vielem unklar, auch in der Gesetzgebung ${ }^{33}$ und in der Rechtswissenschaft. Lediglich einer der o.g. Begriffe existiert in der Verfassung des Staates: „Verwaltungsrechtspflege“ (ma'muriy sud ishlarni yuritish) ${ }^{34}$.

Da die bisherige Entwicklung der usbekischen Gesetzgebung zum Verwaltungsrecht und Verwaltungsprozessrecht sich stets auf verfassungsrechtlichen Vorgaben gründete, lässt sich prognostizieren, dass weitere Verfassungsänderungen wahrscheinlich sind. Es handelt sich dabei um grundlegende Bestimmungen zu den verschiedenen Gerichtsbarkeiten und den Grundprinzipien der Tätigkeit der Verwaltung (insbesondere hinsichtlich des Erlasses von Verwaltungsakten) sowie um die verfassungsrechtlichen Grundlagen der Ahndung von Ordnungswidrigkeiten durch die Gerichte.

\section{c) Tragende Prinzipien eines rechtsstaatlichen Anforderungen entsprechenden Verwaltungsprozesses}

Die genaue methodisch-dogmatische Herleitung der allgemeinen Prinzipien des Verwaltungsrechts sowie damit verbunden des Verwaltungsprozessrechts ist

in allen öffentlich-rechtlichen Streitigkeiten zuständig sein. Dies setzt voraus, dass der geltend gemachte Anspruch auf Öffentliches Recht gestützt wird. Die Organisation der Gerichte und die Besetzung der Spruchkörper stehen im Spannungsverhältnis zwischen der Unabhängigkeit der Richter, einer optimalen Ausstattung für eine bestmögliche Rechtsprechung und der wirtschaftlichen Belastung des Staatshaushalts durch die Ausgaben für die Justiz. Die Vorteile eigenständiger Verwaltungsgerichte liegen in einer starken Spezialisierung der Richter. Dies wirkt sich positiv auf die Bearbeitungszeit und die Sachkunde aus.

Siehe: Pudelka, J. (ed.) (2014). Administrative Process Code: conception, model law and commentary. Berlin: BWV, pp. 12 [Verwaltungsprozesskodex: Konzeption, Modellgesetz und Kommentierung / J. Pudelka (Hrsg.). Berlin: BWV, 2014. 205 S.]

${ }^{32}$ Gleiche Probleme gibt es auch in anderen zentralasiatischen Länder und Russland. Siehe beispielsweise:

Pudelka, J., Deppe, J. (2017). General administrative law in development in Central Asia. Ost/Mag, pp. 19. URL: https://www.ostinstitut.de/documents/publikationen/Pudelka Deppe_Allgemeines_Verwaltungsrecht_in_Zentralasien_in_der_Entwicklung_OL_2_2017.

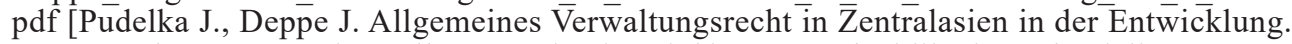
2017. Ost/Mag. URL: https://www.ostinstitut.de/documents/publikationen/Pudelka_Deppe_ Allgemeines_Verwaltungsrecht_in_Zentralasien_in_der_Entwicklung_OL_2_2017.pdf].

Starilov, Yu.N. (1998). Administrative justice in Russia: problems of modern theory and development prospects. Eastern European Law, no. 3-4, pp. 217-252 [Старилов Ю.Н. Административная юстиция в России: проблемы современной теории и перспективы развития. Восточноевропейское право. 1998. № 3-4. С. 217-252].

${ }^{33} \mathrm{Im}$ Gesetzbuch der Republik Usbekistan über die Verwaltungsrechtspflege wurde deshalb die Figur des Verwaltungsaktes nicht berücksichtig, obwohl er meistens der Gegenstand des Verwaltungsprozesses ist.

${ }^{34}$ Vgl. Art. 110 Abs. 1 Verfassung der Republik Usbekistan: „mamuriy sud ishlarini yuritish“. 
sowohl für die Systembildung des Verwaltungsrechts als auch der Rechtsordnung im Allgemeinen von besonderer Bedeutung ${ }^{35}$. Es handelt sich dabei in erster Linie um die Konkretisierung rechtsstaatlicher Gebote $^{36}$. In der nationalen usbekischen Rechtswissenschaft wurden allgemeine Grundsätze eines rechtsstaatlichen Anforderungen entsprechenden Verwaltungsprozesses immer noch nicht formuliert. Aus diesem Grund haben im usbekischen Gesetzbuch über die Verwaltungsrechtspflege die Prinzipien der Zivilprozessordnung (fortan - ZPO) ${ }^{37}$, darunter der dem Verwaltungsprozess fremde Beibringungsgrundsatz, Eingang gefunden ${ }^{38}$. Dabei legte der Gesetzgeber kein Augenmerk auf den für den Verwaltungsprozess relevanten Amtsermittlungsgrundsatz, obgleich gerade das Verwaltungsgericht, anders als das zuständige Gericht in Zivilprozessen, nicht an das Vorbringen der Verfahrensbeteiligten gebunden und nicht auf dieses beschränkt ist ${ }^{39}$. Es muss vielmehr selbst den entscheidungserheblichen Sachverhalt aufklären ${ }^{40}$. Darüber hinaus trägt das Erfordernis der Erforschung des Sachverhalts von Amts wegen dem Grundsatz der Gesetzmäßigkeit der Verwaltung Rechnung; danach besteht ein öffentliches Interesse an der sachlichen Richtigkeit einer behördlichen Entscheidung und einer sie überprüfenden gerichtlichen Entscheidung.

Es ist anzunehmen, dass derzeit eine der wichtigsten Aufgaben der usbekischen Rechtswissenschaft und Rechtsprechung die Identifizierung verbindender Rechtsprinzipien ist. Dies wird zur allgemeinen Stabilisierung der Rechtsordnung beitragen $^{41}$. In diesem Zusammenhang sollte für die nationale Rechtsordnung die aktuelle Erfahrung der Herleitung gemeinsamer Grundprinzipien im Verwaltungsrecht

${ }^{35}$ Sommermann, K.-P. (2014). Principles of administrative law. Bogdandy, A., Cassese, S., Huber, P.M. (eds.). Administrative law in Europe: basic moves. Heidelberg, pp. 864 f, $871 \mathrm{ff}$ [Sommermann K.-P. Prinzipien des Verwaltungsrechts. Verwaltungsrecht in Europa: Gründzüge / A. Bogdandy, S. Cassese, P.M. Huber (Hrsg.). Heidelberg, 2014. S. 864 f, 871 ff].

${ }^{36}$ Sommermann, K.-P. (2014). Principles of administrative law. Bogdandy, A., Cassese, S., Huber, P.M. (eds.). Administrative law in Europe: basic moves. Heidelberg, pp. 874 [Sommermann K.-P. Prinzipien des Verwaltungsrechts. Verwaltungsrecht in Europa: Gründzüge / A. Bogdandy, S. Cassese, P.M. Huber (Hrsg.). Heidelberg, 2014. S. 864 f., 871 ff].

${ }^{37}$ Außer dem sog. Prinzip „Aufklärung des gültigen Sachverhalts sowie der Rechte und Pflichten der Parteiendurch das Gericht", den im Art. 15 ZPO verankert wurde. Siehe die Zivilprozessordnung der Republik Usbekistan vom 22.01.2018 (zuletzt geändert am 12.10.2018). URL: http://lex.uz/docs/3517334.

${ }^{38} \mathrm{Vgl}$. Art. 7-16 ZPO und Art. 8-16 des Gesetzbuches über die Verwaltungsrechtspflege.

${ }^{39}$ Pudelka, J. (ed.) (2014). Administrative Process Code: conception, model law and commentary. Berlin: BWV, pp. 50 f [Verwaltungsprozesskodex: Konzeption, Modellgesetz und Kommentierung / J. Pudelka (Hrsg.). Berlin: BWV, 2014. 205 S.]

${ }^{40}$ Pudelka, J. (ed.) (2014). Administrative Process Code: conception, model law and commentary. Berlin: BWV, pp. 50 f [Verwaltungsprozesskodex: Konzeption, Modellgesetz und Kommentierung / J. Pudelka (Hrsg.). Berlin: BWV, 2014. 205 S.]

${ }^{41}$ Sommermann, K.-P. (2014). Principles of administrative law. Bogdandy, A., Cassese, S., Huber, P.M. (eds.). Administrative law in Europe: basic moves. Heidelberg, pp. $864 \mathrm{ff}$ [Sommermann K.-P. Prinzipien des Verwaltungsrechts. Verwaltungsrecht in Europa: Gründzüge / A. Bogdandy, S. Cassese, P.M. Huber (Hrsg.). Heidelberg, 2014. S. 864 f., 871 ff]. 
europäischer Staaten und daraus folgende Konvergenzen bis hin zur Entwicklung einer europäischen Verwaltungskultur von besonderem Interesse $\operatorname{sein}^{42}$.

\section{d) Verwaltungsgerichtsbarkeit als wichtige Einrichtung im öffentlichen Interesse}

Das Verwaltungsrecht ist ein Rechtsgebiet, dessen Hauptaufgabe die rechtliche Regulierung der gesellschaftlichen Beziehungen im Bereich der Exekutive ist. Im Rahmen dieses Bereichs gilt es, die Umsetzung und den Schutz der Grundrechte der Bürger als auch die Erfüllung der Verpflichtungen durch die Bürger in nahezu allen Bereichen der Gesellschaft zu gewährleisten. Um die Probleme zu lösen, die die Modernisierung der politischen, ökonomischen und sozialen Beziehungen mit sich bringt, ist der staatliche Verwaltungsapparat gezwungen, die Vektoren seiner Tätigkeit neu auszurichten. Dies wiederum führt zu neuen Ansätzen der administrativen und rechtlichen Regulierung der Normsetzungs-, Kontroll- und Aufsichts- sowie anderen Verwaltungsfunktionen, unter anderem zur Gewährleistung des Gleichgewichts zwischen öffentlichen und privaten Interessen in allen Bereichen der Verwaltung. Echte demokratische Werte und die Umsetzung rechtsstaatlicher Prinzipien sollen die Beziehungen zwischen der Gesellschaft, dem Staat und dem Individuum verändern. Somit wachsen die Rolle und die Bedeutung des öffentlichen Interesses, welches im Grunde die gesellschaftlichen Interessen darstellt, ohne deren Befriedigung es unmöglich ist, individuelle Interessen zu verwirklichen und damit ein stabiles Funktionieren des Staates, der Gesellschaft und aller seiner sozialen Schichten zu erreichen.

Aktuelle Bedeutung in diesem Zusammenhang erlangt die Aufgabe der Verbesserung der Funktionsweise der Justiz hinsichtlich der Gewährleistung der gerichtlichen Kontrolle über die Tätigkeit der Verwaltung. Es geht dabei um die Entwicklung des Verwaltungsprozessrechts und seines organischen Bestandteils - nämlich der Verwaltungsgerichtsbarkeit - als wichtige Einrichtung, die zumindest auch öffentlichen Interessen $\mathrm{zu}$ dienen bestimmt ist.

In Usbekistan beruhen die rechtstheoretischen Darstellungen zum öffentlichen Interesse immer noch auf aus sozialistischer Zeit stammendem Formalismus und Positivismus. Insbesondere wird die Konstituierung der Kategorien Öffentliches Recht und öffentliches Interesse durch staatliches Recht und staatliches Interesse aufrechterhalten. Immer noch bestehen eine unklare Trennung zwischen privatem und staatlichem Recht bzw. eine Schwäche des Begriffs des öffentlichen Interesses und eine schwache Wirkung der Verfassung und ihrer Grundsätze ${ }^{43}$. Da die Inhalte des öffentlichen und gemeinen Wohls des sowjetischen Regimes, die in der Regel auf der marxistisch-leninistischen Ideologie basierten, im usbekischen Öffentlichen Recht und seiner Wissenschaft teilweise

${ }^{42}$ Sommermann, K.-P. (2014). Principles of administrative law. Bogdandy, A., Cassese, S., Huber, P.M. (eds.). Administrative law in Europe: basic moves. Heidelberg, pp. $864 \mathrm{f}, 871 \mathrm{ff}$ [Sommermann K.-P. Prinzipien des Verwaltungsrechts. Verwaltungsrecht in Europa: Gründzüge/ A. Bogdandy, S. Cassese, P.M. Huber (Hrsg.). Heidelberg, 2014. S. 864 f., 871 ff].

${ }^{43}$ Pudelka, J., Deppe, J. (2017). General administrative law in development in Central Asia. Ost/Mag, pp. 5. URL: https://www.ostinstitut.de/documents/publikationen/Pudelka Deppe Allgemeines_Verwaltungsrecht_in_Zentralasien_in_der_Entwicklung_OL_2_2017.pdf [Pudelka J., Deppe J. Allgemeines Verwaltungsrecht in Zentralasien in der Entwicklung. 2017. Ost/Mag. URL: https://www.ostinstitut.de/documents/publikationen/Pudelka_Deppe_Allgemeines_ Verwaltungsrecht_in_Zentralasien_in_der_Entwicklung_OL_2_2017.pdf]. 
immer noch Wirkung entfalten, verhindern sie die Entstehung eines Verständnisses des öffentlichen Interesses auf der Basis einer demokratischen Verfassungstheorie und Lehre der öffentlichen Gewalt.

In der Rechtswissenschaft Usbekistans (und ebenso anderer postsowjetischer Länder $)^{44}$ sind die Meinungen über die Beziehung zwischen solchen Begriffen wie „öffentliches“, „staatliches“ und „gesellschaftliches“ Interesse oder Interesse eines „unbestimmten Personenkreises“ noch mehrdeutig: von ihrer Unterscheidung bis zur vollständigen Gleichsetzung. Gleichzeitig hat sich die Wahrnehmung des Verfahrensinstituts des Schutzes des öffentlichen Interesses noch nicht entwickelt, weder auf der Ebene der Rechtstheorie noch im Prozessrecht. So enthalten die verschiedenen Rechtswege der Verwaltungsgerichtsbarkeit in Usbekistan keine Mechanismen zur Einbringung des öffentlichen Interesses in den Verwaltungsprozess. Insbesondere taucht der Begriff des öffentlichen Interesses in verwaltungsprozessrechtlichen Normen nicht auf, obwohl die Verwaltungsgerichte die beschränkte Befugnis haben, im Streitfall die Auslegung und Anwendung des ,, staatlichen und gesellschaftlichen “ Interesses durch die Verwaltung auf ihre Rechtmäßigkeit zu überprüfen. So spielt in der Rechtsordnung die Staatsanwaltschaft (auch noch eine Reihe weiterer Staatsorgane, u.a. das Justizministerium, die Ombudsbehörde) als Vertreterin des öffentlichen Interesses im Verwaltungsprozess eine bedeutende Rolle ${ }^{45}$. Dabei existieren keine Methoden und Techniken richterlicher Entscheidungsfindung, die öffentlichen Interessen zu determinieren und damit im Urteil bzw. Beschluss zu rechtlichen öffentlichen Interessen zu machen.

Die Durchführung einer vertieften wissenschaftlichen Untersuchung, die das öffentliche Interesse im Verwaltungsrecht zum Inhalt hat, kann Grundlagen für weitere Forschungen zur Begleitung der Reform des Öffentlichen Rechts legen. Gleichzeitig ist

${ }^{44}$ So wurden in der russischen Rechtswissenschaft die Grundlagen für die Erforschung der Kategorie des „öffentlichen Interesses“ in den 1990er Jahren gelegt, bis im Jahr 2003 der Begriff Eingang in die Zivilprozessordnung fand. Siehe beispielweise: Tichomirov, Yu.A. (1995). Public law. Moscow [Тихомиров Ю.А. Публичное право. Москва, 1995. 496 с.].

Ju.A. Tichomirov definiert das öffentliche Interesse als ,ein vom Staat anerkanntes und durch das Recht gewährleistete Interesse der sozialen Gemeinschaft, dessen Befriedigung als Garantie für ihre Existenz und Entwicklung dient".

${ }^{45}$ Betreffs der Zuständigkeit der Staatsanwaltschaft siehe nur Art. 46 Gesetzbuch der Republik Usbekistan über die Verwaltungsrechtspflege. Die deutschen Experten gehen davon aus, dass die Staatsanwaltschaft derzeit nicht ausgeschlossen werden kann, sondern sie so einzubinden sei, dass der verwaltungsrechtliche Individualrechtsschutz gestärkt und stellenweise erweitert wird, andererseits allerdings eine allgemeine Rechtmäßigkeitsaufsicht abgelehnt wird. Allerdings wird in der „Konzeption zur Einführung eines Verwaltungsprozesskodexes“ eine allgemeine Rechtmäßigkeitsaufsicht abgelehnt.

Siehe: Pudelka, J., Deppe, J. (2017). General administrative law in development in Central Asia. Ost/Mag, pp. 24. URL: https://www.ostinstitut.de/documents/publikationen/Pudelka Deppe_Allgemeines_Verwaltungsrecht_in_Zentralasien_in_der_Entwicklung_OL_2_2017.pdf [Pudelka J., Deppe J. Āllgemeines Verwaltungsrecht in Zentralasien in der Entwicklung. 2017 . Ost/ Mag. URL: https://www.ostinstitut.de/documents/publikationen/Pudelka_Deppe_Allgemeines Verwaltungsrecht_in_Zentralasien_in_der_Entwicklung_OL_2_2017.pdf].

Pudelka, J. (ed.) (2014). Administrative Process Code: conception, model law and commentary. Berlin: BWV [Verwaltungsprozesskodex: Konzeption, Modellgesetz und Kommentierung / J. Pudelka (Hrsg.). Berlin: BWV, 2014. 205 S.]. 
es angesichts der im Zuge der Globalisierung zunehmenden Transformationsprozesse der nationalen Rechtssysteme ratsam, solche wissenschaftlichen Ausarbeitungen auf der Grundlage rechtsvergleichender Untersuchungen unter Berücksichtigung der Erfahrungen anderer Staaten, die bedeutende Ergebnisse in der Entwicklung der Rechtsstaatlichkeit und etablierte wissenschaftliche Schulen des Öffentlichen Rechts vorweisen können sowie allgemeine Merkmale des Rechtssystems gemeinsam haben, aufzubauen.

Ausgehend von den Ergebnissen einer vorläufigen Analyse der vorherrschenden doktrinellen Ansätze deutscher Rechtswissenschaftler ${ }^{46}$ in diesem Bereich wie auch der etablierten Praxis der Rechtsanwendung in Deutschland, wo es seit 1945 viele Versuche gab, das öffentliche Interesse als juristische Kategorie allgemein zu erfassen, ist für das Rechtssystem Usbekistans gerade die deutsche Erfahrung besonders wertvoll und nützlich.

\section{Relevante gesellschaftliche, politische und rechtliche Bedingungen für die Akzeptanz der Verwaltungsgerichtsbarkeit}

Die Zeit seit der Unabhängigkeit Usbekistans ist durch die Einführung von Organisationsformen und Prinzipien westlicher Regierungsführung gekennzeichnet, die von Pragmatismus, universellen formalen Regeln und Rechtsnormen als Hauptgrundlagen für Handlungen und Entscheidungen dominiert werden. In die Tätigkeit der politischen Institutionen bleiben gleichzeitig das niedrige Niveau der Universalisierung des Rechts, Doppeldenk auf der Mikroebene der modernen Praktiken ${ }^{47}$ und der Konflikt der moralischen Anschauungen in der modernen Gesellschaft mit paternalistischem Bewusstsein und paternalistischen Beziehungen erhalten. Darüber hinaus ist in letzter Zeit ein zunehmender Einfluss der Religion auf die Organisation der öffentlichen Verwaltung zu verzeichnen ${ }^{48}$. Gleichzeitig ist es ermutigend, dass die neue Staatsführung

${ }^{46}$ Haberle, P. (2006). Public interest as a legal problem: an analysis of legislation and case law. 2nd ed. Berlin [Häberle P. Öffentliches Interesse als juristisches Problem: Eine Analyse von Gesetzgebung und Rechtsprechung. 2. Aufl. Berlin, 2006. 700 S.].

Uerpmann, R. (1999). The public interest: its meaning as an element of fact and as a dogmatic concept. Tubingen [Uerpmann R. Das öffentliche Interesse - Seine Bedeutung als Tatbestandsmerkmall und als dogmatischer Begriff. Tübingen, 1999. 420 S.].

Masing, J. (1997). Mobilizing the burgers for law enforcement: European impulses for a revision of the teaching of subjective public law. Berlin [Masing J. Die Mobilisierung des Burgers für die Durchsetzung des Rechts: europäische Impulse für eine Revision der Lehre vom subjektiv-öffentlichen Recht. Berlin, 1997. 288 S.].

Nolte, J. (2015). The peculiarity of administrative judicial protection. Reason and limits of the application of civil procedure law in the administrative process. Berlin [Nolte J. Die Eigenart des verwaltungsgerichtlichen Rechtschutzes. Grund und grenzen der Anwendung des Zivilprozessrechts im Verwaltungsprozess. Berlin, 2015. 678 S.].

${ }^{47}$ Das heißt, dass das Gesetz und die formalen Regeln bekannt, jedoch kein Wertbestandteil des Verhaltens und kein wesentliches Handlungsmotiv sind.

${ }^{48}$ In Usbekistan ist die Gedankenfreiheit in Art. 31 der Verfassung, die Trennung von Staat und Religion in Art. 5 des Gesetzes der Republik Usbekistan „Über Gedankenfreiheit und religiösen Glaubensgemeinschaften" festgeschrieben. Die staatlich-konfessionellen Beziehungen im Land sind jedoch heute durch zwei einander gegenläufige Tendenzen gekennzeichnet. Einerseits werden durch die Globalisierung des öffentlichen Lebens sowie durch die Umsetzung von Verwaltungsund sozioökonomischen Reformen in Usbekistan die Werte der westlichen Demokratie gefestigt, 
den Wunsch nach Entwicklung einer liberalen Ideologie, einer sozialen Marktwirtschaft, eines freien Marktes, demokratischer politischer Institutionen und der Voraussetzungen für die Schaffung eines Rechtsstaats in Usbekistan zum Ausdruck bringt.

\section{a) Das Wesen der Verwaltungsreform als Systementscheidung}

Die Implementierung rechtsstaatlicher Prinzipien in Wirtschaft, Politik und Gesellschaft sowie die Befähigung der Justiz, entsprechende Normen anzuwenden, sind wichtige Ziele der Reformprozesse in Usbekistan. Das Regierungshandeln gewann an Transparenz. In Usbekistan laufen zurzeit intensive Prozesse zur Schaffung eines vollkommen neuen und effektiv funktionierenden Systems der Staatsverwaltung. Zudem werden neue Rechtsgrundlagen über den Staatsdienst ausgearbeitet ${ }^{49}$.

Im Land wurde bis heute viel getan, um das System der Staatsverwaltung grundlegend $\mathrm{zu}$ verbessern und zu modernisieren. Dennoch hat eine objektive Beurteilung der im Laufe der letzten zwei Jahre durchgeführten Maßnahmen und der Ergebnisse der Tätigkeit von Organen der Staatsverwaltung sowie der offene und direkte Dialog mit der Bevölkerung einen Bedarf an einer systemhaften Lösung bestehender Probleme und Mängel ergeben. Dies betrifft in erster Linie: den rein deklarativen Charakter der Aufgaben einiger Ressorts und die mangelhaften organisationsrechtlichen Voraussetzungen ihrer Erfüllung; ein ineffektives System der Leistungsbeurteilung, die lediglich auf die Feststellung von Tatsachen und das Sammeln von Statistiken hinausläuft und häufig den tatsächlichen Stand der Dinge vor Ort nicht wiedergibt; die übermäßige Zentralisierung staatlicher Funktionen, die die Rolle der sog. Örtlichen Organe der Staatsmacht (staatliche Verwaltung in den Gebietskörperschaften) bei der Gestaltung von territorialen Entwicklungsprogrammen und bei der Bewältigung dringender Probleme der Bevölkerung verringert; ein niedriges Niveau der innovativen Entwicklung, das zu Bürokratisierung und hohem Aufwand führt; Kombinierung von staatlichen regulativen und wirtschaftlichen Funktionen durch Organe der

die wichtigsten Institutionen der Zivilgesellschaft gebildet und die liberalen Ideen der autonomen Persönlichkeit eingeführt. Andererseits verstärkt sich das religiöse Bewusstsein der Bevölkerung, vor allem der muslimischen Mehrheit, und der Einfluss der islamischen Religion auf den sozialen und politischen Bereich der Gesellschaft nimmt zu. Zum Beispiel berichteten die Medien unlängst über die Organisation einer Zeremonie, wo Polizeibeamte eines Landkreises des Taschkenter Gebietes einen Eid auf den Koran ablegen und dabei versprechen, keine Korruptionsdelikte, vor allem nicht den Straftatbestand der Bestechlichkeit, zu verwirklichen. An dieser Zeremonie nahm auch der Hauptimam des Landkreises teil. Siehe Radio Ozodlik, URL: https://rus.ozodlik. org/a/29644714.html und auch die russischsprachige Internetzeitung Versja, URL: https:// versia.ru/uzbekskie-milicionery-poklyalis-na-korane-ne-brat-vzyatki (zuletzt abgerufen am 28.12.2018). Besagte Tendenzen stellen besondere Aufgaben für die Rechtswissenschaft. Es erscheint notwendig, ein neues wissenschaftlich fundiertes Modell der staatlich-konfessionellen Beziehungen $\mathrm{zu}$ entwickeln und umzusetzen. Dieses Modell sollte zum Ziel haben, die verfassungsrechtlichen Prinzipien des Säkularismus detailliert in Gesetzen umzusetzen, die gemäß den Anforderungen des demokratischen Rechtsstaates das Verhältnis zwischen Staat und religiösen Institutionen festlegen werden. Gleichzeitig wird die Festlegung rechtlicher Grenzen für die Annäherung von Macht und Religion auch dazu beitragen, einen übermäßigen Einfluss der Religion auf die Staatsverwaltung zu verhindern.

${ }^{49}$ Vgl. Erlass des Präsidenten Republik Usbekistan „Über die Bestätigung der Konzeption der Verwaltungsreform in der Republik Usbekistan“" vom 08.09.2017 № PF-5185. URL: http://old.lex.uz/pages/getpageaspx?lact_id=3331176. 
Wirtschaftsverwaltung, die Praxis einer wahlweisen Bereitstellung von Vergünstigungen und Präferenzen, die die Entwicklung eines gesunden Wettbewerbsumfelds behindern; und fehlende pflichtgemäße Verantwortung und Initiative bei einigen Führungskräften, was sich negativ auf die territoriale Entwicklung auswirkt ${ }^{50}$. Diese Mängel behindern eine erfolgreiche Modernisierung der Wirtschaftsbranchen und des sozialen Bereichs, eine umfassende territoriale Entwicklung sowie die Steigerung des Lebensstandards der Bevölkerung ${ }^{51}$.

In diesem Zusammenhang wurde vom Präsidenten der Republik Usbekistan im September 2017 der Erlass „Über die Bestätigung der Konzeption der Verwaltungsreform in der Republik Usbekistan“ (PF-5185) ${ }^{52}$ verabschiedet, der einen wichtigen Schritt zur Umsetzung der Handlungsstrategien in den fünf Schwerpunktbereichen der Entwicklung der Republik Usbekistan in den Jahren 2017-202153 darstellt und einen Umbruch in Staat und Gesellschaft Usbekistans markiert. In der Konzeption wurden sechs Schwerpunktbereiche der grundlegenden Reform des Systems der Staatsverwaltung definiert: Verbesserung der institutionellen und organisationsrechtlichen Grundlagen der Tätigkeit der Verwaltung (I.); Konkretisierung von Aufgaben (Funktionen, Befugnissen), Mechanismen ihrer Erfüllung und der Zuständigkeitsbereiche der Verwaltung, Verbesserung der Prozesse der Koordinierung als auch der Kooperation (II.); Weiterer Abbau der Einflussnahme der Verwaltung auf einzelne Wirtschaftsbranchen und Ausbau der marktwirtschaftlichen Steuerungsmechanismen (III.); Verbesserung der Mechanismen des vertikalen Steuerungssystems und der Kooperation der Staatsverwaltung (IV.); Einführung moderner Formen der strategischen Planung, innovativer Ideen, Entwicklungen und Technologien im System der Staatsverwaltung (V.) und Gestaltung eines effektiven Systems des professionellen öffentlichen Dienstes, Einführung wirksamer Mechanismen der Korruptionsbekämpfung im System der Staatsverwaltung (VI.).

Durch diese Verwaltungsreform soll eine Reihe von Ergebnissen erzielt werden, unter anderem die Schaffung wirksamer Mechanismen zur Gewährleistung der Rechte und Freiheiten der Bürger, eine weitere Steigerung ihres Wohlstands und des Grads der Zufriedenheit mit der Tätigkeit der Staatsverwaltung, insbesondere durch:

- eine Verbesserung der Verwaltungsverfahren, die auf eine klare Regelung der Rechtsbeziehun gen der Staatsorgane mit natürlichen und juristischen Personen gerichtet sind;

${ }^{50}$ Vgl. Erlass des Präsidenten Republik Usbekistan „Über die Bestätigung der Konzeption der Verwaltungsreform in der Republik Usbekistan“" vom 08.09.2017 № PF-5185. URL: http://old.lex.uz/pages/getpageaspx?lact_id=3331176.

${ }^{51}$ Vgl. Erlass des Präsidenten Republik Usbekistan „Über die Bestätigung der Konzeption der Verwaltungsreform in der Republik Usbekistan“" vom 08.09.2017 № PF-5185. URL: http://old.lex.uz/pages/getpageaspx?lact_id=3331176.

${ }^{52}$ Vgl. Erlass des Präsidenten Republik Usbekistan „Über die Bestätigung der Konzeption der Verwaltungsreform in der Republik Usbekistan“" vom 08.09.2017 № PF-5185. URL: http://old.lex.uz/pages/getpageaspx?lact_id=3331176.

${ }^{53}$ Siehe: Erlass des Präsidenten der Republik Usbekistan, Über Handlungsstrategien in den fünf Schwerpunktbereichen der Entwicklung der Republik Usbekistan in den Jahren 2017-2021" vom 07.02.2017 № PF-4947. URL: http://old.lex.uz/pages/getpage.aspx?lact_id=3107042\#3109624. 
- die Entwicklung des Systems der Verwaltungsjustiz, einschließlich einer Verbesserung des Verfahrens zur Anfechtung der Entscheidungen und des Handelns der Staatsverwaltung, der Einführung von Mechanismen der kollegialen Anhörung von Eingaben natürlicher und juristischer Personen;

- $\quad$ eine Steigerung der Effizienz der Erbringung öffentlicher Dienstleistungen durch Verbesserung des E-Government-Systems;

- Gewährleistung der Rechtsstaatlichkeit, Stärkung der Gesetzlichkeit und der Rechtsordnung in der Gesellschaft.

Die Umsetzung der in der Konzeption der Verwaltungsreform genannten Maßnahmen verleiht der Modernisierung des Systems der Staatsverwaltung unter Berücksichtigung moderner Tendenzen der innovativen Entwicklung und der internationalen Standards einen neuen Impuls. Wie in der Konzeption explizit vorgesehen ist, soll die Umsetzung der Verwaltungsreform zur Realisierung folgender Idee beitragen: „, Nicht das Volk soll der Staatsverwaltung, sondern die Staatsverwaltung dem Volke dienen "54.

Ausgehend von der Analyse der Bestimmungen der Konzeption kann man zu dem Schluss kommen, dass der Staat die Notwendigkeit grundlegender Veränderungen in Organisation und Aufbau der öffentlichen Verwaltung begriffen hat. Alle mehr oder weniger wesentlichen Aspekte der Probleme im Bereich des Öffentlichen Rechts wurden in der Konzeption angesprochen, insbesondere: Wer verwaltet, wie, zu welchem Zweck, mit welchem Nutzeffekt. Die schwierigste und delikateste Aufgabe der begonnenen Reformen besteht jedoch darin, die Beschaffenheit und die Methoden der Verwaltung zu verändern, ihnen Eigenschaften zu geben, die den allgemein anerkannten demokratischen und rechtsstaatlichen Prinzipien entsprechen. Die erfolgreiche Umsetzung dieser Aufgabe wird conditio sine qua non für mehr Akzeptanz der Verwaltungsgerichtsbarkeit sein.

\section{b) Rolle des Rechts in der rechtsstaatlichen Modernisierung des Staates}

Die gegenwärtige Etappe der gesellschaftlichen Entwicklung der Republik Usbekistan, die vor allem mit dem Aufbau eines demokratischen Rechtsstaates und der Zivilgesellschaft verbunden ist, lässt sich durch eine stärkere Rolle des Rechts und durch die Einbeziehung von immer mehr Menschen in rechtliche Verhältnisse charakterisieren. Der objektive Prozess der Stärkung der Rolle des Rechts bei der Regelung der gesellschaftlichen Verhältnisse, des Verhaltens von sozialen Gruppen und Personen lässt sich wiederum darauf zurückführen, dass das Recht in Usbekistan ein Werkzeug für die Äußerung und Verwirklichung des Volkes ist ${ }^{55}$. In diesem Sinne stellt das Recht nicht nur eine Gesamtheit von juristischen Normen, sondern auch ein kompliziertes dynamisches Sozialsystem dar, das imstande ist, die Regelungsfunktion der

\footnotetext{
${ }^{54}$ Siehe Erlass des Präsidenten der Republik Usbekistan vom 08.09.2018 № PF-5185.

${ }^{55}$ Egamberdiev, A. (2007). Results of the seminar on "From the Khanate to the Republic. The 20th century: structures and basic values in the field of Uzbekistan". Krumm, R., Gunther, Ch., Klingenberg, M., Ryssel, G. (eds.). History and Identity II: Uzbekistan and Germany in the XX. century. Taschkent, pp. 145 f [Egamberdiev A. Ergebnisse des Seminars zum Thema „Vom Khanat zur Republik. Das 20. Jahrhundert: Strukturen und Grundwerte auf dem Gebiet Usbekistans“. Geschichte und Identität II: Usbekistan und Deutschland im XX. Jahrhundert / R. Krumm, Ch. Günther, M. Klingenberg, G. Ryssel (Hrsg.). Taschkent, 2007. S. 145 f].
} 
gesellschaftlichen Verhältnisse sowie die Funktion einer wissenschaftlich begründeten Steuerung unterschiedlicher gesellschaftlicher Prozesse zu erfüllen.

Usbekistan absolviert deshalb immer noch einen Transformationsprozess, dessen Ziel es sein muss, nationale Traditionen mit der rechtsstaatlichen Modernisierung des Staats in Einklang zu bringen. Mit den Transformationsprozessen in der nationalen Rechtswissenschaft (wie übrigens auch in den meisten postsowjetischen Ländern) geht zugleich ein Prozess der vielaspektischen Umdeutung von Staat und Recht sowie ihrer Wechselwirkung einher, insbesondere auch zwischen Privatem und Öffentlichem Recht sowie der Wirkung der Verfassung und ihrer Grundsätze. Die Verfassungsaufgabe, einen humanen und demokratischen Rechtsstaat zu schaffen, impliziert eine den demokratischen Prinzipien entsprechende Organisationsform der öffentlichen Verwaltung. Ähnlich wie sich zurzeit die Beschaffenheit und die Methoden der öffentlichen Verwaltung verändern, deutet sich eine Erneuerung der Strategie und der Methoden rechtlicher Regulierung der gesellschaftlichen Beziehungen an, was insbesondere einen Übergang von der Verwaltung eines „Kommandostaates“ zur Verwaltung von Interessen und durch Interessen beinhaltet. Entsprechend dieser Aufgabe muss man die theoretische Vorstellung über das Verhältnis von politischer Gewalt und Recht sowie von Politik und Recht im Allgemeinen ändern.

Sicher ist einerseits der Wille der Transformationsstaaten unübersehbar, das sowjetische Erbe über Bord zu werfen ${ }^{56}$. Viele Gesetze des Öffentlichen Rechts sind inzwischen modernisiert worden oder befinden sich in gesetzgeberischer Bearbeitung und Diskussion $^{57}$. Aber andererseits sind die Schwierigkeiten unverkennbar, rechtsstaatliche und demokratische Grundsätze anzunehmen und zu implementieren ${ }^{58}$. Ausgehend davon ist es notwendig, das in Usbekistan derzeit noch vorherrschende Verständnis von Recht im Ganzen zu verändern. Leider ist bisher die Forschung zur Wechselwirkung zwischen Staat und Recht im Land kaum spürbar. In Ergebnis ist es - sowohl in der nationalen Fachliteratur als auch in der Praxis - immer noch verbreitet, das Recht auf das Gesetz zu reduzieren. Dies ist aber kontraproduktiv, da die inhaltliche Qualität eines Gesetzes ausmacht, dass es den öffentlichen Bedürfnissen entspricht und ein gewisses

${ }^{56}$ Pudelka, J., Deppe, J. (2017). General administrative law in development in Central Asia. Ost/Mag, pp. 2-3. URL: https://www.ostinstitut.de/documents/publikationen/Pudelka_Deppe Allgemeines Verwaltungsrecht in Zentralasien in der_Entwicklung_OL_2 2017.pdf [Pudelka J., Deppe J. Allgemeines Verwaltungsrecht in Zentralasien in der Entwicklung. 2017. Ost/Mag. URL: https://www.ostinstitut.de/documents/publikationen/Pudelka_Deppe_Allgemeines_ Verwaltungsrecht_in_Zentralasien_in_der_Entwicklung_OL_2_2017.pdf].

${ }^{57}$ Pudelka, J., Deppe, J. (2017). General administrative law in development in Central Asia. Ost/Mag, pp. 2-3. URL: https://www.ostinstitut.de/documents/publikationen/Pudelka_Deppe Allgemeines_Verwaltungsrecht_in_Zentralasien_in_der_Entwicklung_OL_2_2017.pdf [Pudelka J., Deppe J. Allgemeines Verwaltungsrecht in Zentralasien in der Entwicklung. 2017. Ost/Mag. URL: https://www.ostinstitut.de/documents/publikationen/Pudelka_Deppe_Allgemeines_ Verwaltungsrecht_in_Zentralasien_in_der_Entwicklung_OL_2_2017.pdf].

${ }^{58}$ Pudelka, J., Deppe, J. (2017). General administrative law in development in Central Asia. Ost/Mag, pp. 2-3. URL: https://www.ostinstitut.de/documents/publikationen/Pudelka_Deppe Allgemeines_Verwaltungsrecht_in_Zentralasien_in_der_Entwicklung_OL_2_2017.pdf [Pudelka J., Deppe J. Allgemeines Verwaltungsrecht in Zentralasien in der Entwicklung. 2017. Ost/Mag. URL: https://www.ostinstitut.de/documents/publikationen/Pudelka_Deppe_Allgemeines_ Verwaltungsrecht_in_Zentralasien_in_der_Entwicklung_OL_2_2017.pdf]. 
$\mathrm{Maß}$ an Freiheit, Gleichheit und Gerechtigkeit gewährleistet, deren Wurzeln bis in den Charakter der gesellschaftlichen Produktionsverhältnisse reichen sollten. Die Idee eines humanen und demokratischen Rechtsstaates beschränkt sich nicht auf Herrschaft des Rechts. Sie setzt eine optimale Annäherung der Rechtsvorschriften an die objektive Rechtsnatur der regulierten Beziehungen voraus, widersetzt sich der legislativen Sanktionierung der Willkür, stellt die Funktionen und die Struktur des Staates auf eine verfassungsrechtliche Grundlage und erhebt - nicht nur mit Worten, sondern mit Taten - zum obersten Ziel staatlicher Politik die Interessen des Volkes und die Rechte und Freiheiten der Bürger. Zum Kriterium des sozialen Fortschritts des Landes bestimmt sie den Grad der Entwicklung des Individuums und seiner kreativen Fähigkeiten.

Die Entwicklung eines modernen Rechtsverständnisses ist nicht einfach, weil es eine tiefe philosophische Einsicht in das Wesen der Dinge erfordert, einen wirklich humanitären Ansatz, die Synthese von natürlichem und sozialem Wissen, die Einbeziehung der Rechtstheorie in den Komplex der Humanwissenschaften. Vor allem erscheint es notwendig, das Demokratieprinzip und Rechtsstaatlichkeitsprinzip in der nationalen Staats- und Rechtstheorie weiterzuentwickeln, d.h. eine Abkehr vom allgegenwärtigen Staatszentrismus zu vollziehen. Dabei sind - aufgrund der Systematik bzw. Terminologie des usbekischen Verfassungsrechts - nicht „Rechtsstaat“ bzw. „, Rule of Law“, sondern „,Vorrang des Gesetzes“ und „, Gesetzmäßigkeit“59 Schlüsselbegriffe für den Weg zu rechtsstaatlichen Verhältnissen in Usbekistan. Es ist aber anzunehmen, dass es kaum möglich ist, unter Beibehaltung der bestehenden traditionellen Vorstellungen und konzeptuellen Ansätze zu Staat und Recht eine moderne und effektive Verwaltungsgerichtsbarkeit aufzubauen.

Das Problem der Rechtsstaatlichkeit ist sehr komplex, und die Entwicklung dieser Doktrin in Usbekistan ist ein sehr schwieriger Prozess, der so organisiert werden muss, dass Rechtsstaatlichkeit nicht als sehr allgemeine und hohe Losung wahrgenommen wird. In keinem Fall sollte Rechtsstaatlichkeit darauf reduziert werden, dass Gesetze eingehalten werden müssen, da sowohl die Qualität der Gesetze als auch die Qualifikation derjenigen, die sie anwenden, nicht immer gut sind. Vorab ist die Frage des Verhältnisses zwischen Rechtsstaatlichkeit, Staat, Wirtschaft und dem Individuum wichtig. Es sind diese Komponenten, die notwendige Bestandteile der Frage sind, und ohne ihre Synthese zu erreichen, ist es unmöglich, weder einen Aktionsplan, eine Strategie oder Vorhersagen zu entwickeln noch die Situation sachgerecht zu analysieren.

Wichtig ist auch die Frage nach einer umfassenden Untersuchung und Entwicklung von Standards, insbesondere von Standards im Bereich der staatlichen Macht und des Schutzes der Person, einschließlich einer eingehenden Analyse einschlägiger Auslandserfahrung. Leider liegen vielen Rechtsvorschriften in den einzelnen Rechtsbereichen oft fehlerhaften Vorstellungen und keine allgemein anerkannten Standards zugrunde. Hier kann man ebenfalls die Erfahrung Deutschlands nutzen, wo als Rechtsstaat eine Ordnung verstanden wird, welche beansprucht, staatliche

${ }^{59}$ Der „Vorrang des Gesetzes“ und die „Gesetzmäßigkeit“ als Rechtsbegriffe gelten in der usbekischen Rechtsordnung immer noch, wie in der Sowjetzeit, als eine zentrale Maxime der Rechtsetzung und Rechtsanwendung. 
Machtbefugnisse nach Maßgabe von Recht und Gerechtigkeit auszuüben ${ }^{60}$. Dementsprechend zeichnet sich die Rechtsstaatlichkeit der Bundesrepublik Deutschland durch eine umfassende rechtliche Bindung der staatlichen Institutionen, Mandatsträger und Amtswalter sowie durch die Gewährleistung der Grundrechte aus ${ }^{61}$. Obwohl der verfassungsrechtliche Standort des Rechtsstaatsprinzips in Deutschland umstritten ist, wird überwiegend das Rechtsstaatsprinzip in Art. 20 Abs. 3 GG verortet gesehen ${ }^{62}$. Das rechtsstaatliche Prinzip des Grundgesetzes hat jedoch eine materielle Dimension und es verlangt nicht nur Gesetzmäßigkeit staatlichen Handelns, sondern verpflichtet auch, das staatliche Handeln auf die Verwirklichung materieller Gerechtigkeit auszurichten ${ }^{63}$. Dies ergibt sich zum einen aus der unmittelbaren Grundrechtsbindung nach Art. 1 Abs. 3 GG, andererseits aus der Bindung an Gesetz und Recht nach Art. 20 Abs. 3 GG $^{64}$.

Ungeachtet einiger Reformbemühungen der letzten Jahre entsprechen bei Weitem noch nicht alle Normativakte rechtsstaatlichen Maßstäben. Außerdem weist die Anwendung der Reformgesetze vielfach Defizite auf. So heben die deutschen Experten hervor, dass es für nachhaltige Veränderungen im Verwaltungsrecht nicht allein auf formale Reformschritte (neue Gesetze, neue Institutionen) ankomme, sondern auf echte Veränderungen, Innovationen, substantielle Reformen und einen Bewusstseinswandel unter den professionellen Rechtsanwendern wie auch in der Bevölkerung ${ }^{65}$. Das Rechtsbewusstsein der Bürger sei hierbei ebenso wichtig wie die Ausbildung und Qualifizierung von Beamten, Juristen und Richtern ${ }^{66}$.

${ }^{60}$ Sodan, H., Ziekow, J. (2018). Basic course in public law, constitutional and administrative law. 8th ed. Berlin, pp. 73, Rndr. 1 [Sodan H., Ziekow J. Grundkurs Öffentliches Recht, Staatsund Verwaltungsrecht. 8. Aufl. Berlin, 2018. S. 737, Rndr. 3]

${ }^{61}$ Sodan, H., Ziekow, J. (2018). Basic course in public law, constitutional and administrative law. 8th ed. Berlin, pp. 737, Rndr. 3 [Sodan H., Ziekow J. Grundkurs Öffentliches Recht, Staatsund Verwaltungsrecht. 8. Aufl. Berlin, 2018. S. 737, Rndr. 3]

${ }^{62}$ Sodan, H., Ziekow, J. (2018). Basic course in public law, constitutional and administrative law. 8th ed. Berlin, pp. 73, Rndr. 2 [Sodan H., Ziekow J. Grundkurs Öffentliches Recht, Staatsund Verwaltungsrecht. 8. Aufl. Berlin, 2018. S. 737, Rndr. 3]

${ }^{63}$ Sodan, H., Ziekow, J. (2018). Basic course in public law, constitutional and administrative law. 8th ed. Berlin, pp. 73, Rndr. 3 f [Sodan H., Ziekow J. Grundkurs Öffentliches Recht, Staatsund Verwaltungsrecht. 8. Aufl. Berlin, 2018. S. 737, Rndr. 3]

${ }^{64}$ Sodan, H., Ziekow, J. (2018). Basic course in public law, constitutional and administrative law. 8th ed. Berlin, pp. 737, Rndr. 3 [Sodan H., Ziekow J. Grundkurs Öffentliches Recht, Staatsund Verwaltungsrecht. 8. Aufl. Berlin, 2018. S. 737, Rndr. 3]

${ }^{65}$ Pudelka, J., Deppe, J. (2017). General administrative law in development in Central Asia. Ost/Mag, pp. 6. URL: https://www.ostinstitut.de/documents/publikationen/Pudelka_Deppe Allgemeines_Verwaltungsrecht_in_Zentralasien_in_der_Entwicklung_OL_2_2017.pdf [Pudelka J., Deppe J. Allgemeines Verwaltungsrecht in Z̄entralasien in der Entwicklung. 2017. Ost/Mag. URL: https://www.ostinstitut.de/documents/publikationen/Pudelka_Deppe_Allgemeines_ Verwaltungsrecht_in_Zentralasien_in_der_Entwicklung_OL_2_2017.pdf].

${ }^{66}$ Pudelka, J., Deppe, J. (2017). General administrative law in development in Central Asia. Ost/Mag, pp. 6. URL: https://www.ostinstitut.de/documents/publikationen/Pudelka_Deppe Allgemeines_Verwaltungsrecht_in_Zentralasien_in_der_Entwicklung_OL_2_2017.pdf [Pudelka J., Deppe J. Allgemeines Verwaltungsrecht in Zentralasien in der Entwicklung. 2017. Ost/Mag. URL: https://www.ostinstitut.de/documents/publikationen/Pudelka_Deppe_Allgemeines_ Verwaltungsrecht_in_Zentralasien_in_der_Entwicklung_OL_2_2017.pdff]. 
BeiderSchaffung eines anDemokratie und Rechtsstaatorientierten Rechtsbewusstseins muss es in erster Linie um die für die rechtsstaatlichen Reformprozesse relevanten Akteure (Beamten der Exekutive, Richter, Staatsanwälte, Rechtsanwälte und Studenten der Rechtswissenschaft) gehen. Aufgrund des immer noch fortwirkenden Einflusses des „sozialistischen Rechtsbewusstseins" haben die meisten dieser Akteure keine klaren Vorstellungen vom Wesen und von den Besonderheiten des Verwaltungsrechtschutzes im Kontext der rechtsstaatlichen Modernisierung des Staates. Die genannten Akteure sind daran gewöhnt, dass die Exekutive nicht von klagenden Bürgern und urteilenden Gerichten, sondern von der Rechtaufsicht durch die Exekutive ${ }^{67}$ selbst bzw. Staatsanwaltschaft kontrolliert wird ${ }^{68}$.

In diesem Zusammenhang kommt der Rechtswissenschaft eine konstitutive Bedeutung zu. Jedoch sind in Usbekistan bisher sowohl Forschung als auch Lehrbücher, die sich mit dem neueren Staats- und Verwaltungsrecht auseinandersetzen, kaum vorhanden ${ }^{69}$. Im Ergebnis bereiten die usbekischen juristischen Fakultäten ihre Studierenden unzureichend auf die Techniken zur Berücksichtigung rechtsstaatlicher Standards, insbesondere der

${ }^{67}$ So wurden Anfang 2017 in allen Regionen des Landes (in der Republik Karakalpakstan, in Taschkent und allen Gebieten, Landkreisen und Stadtbezirken, insgesamt in 207 Siedlungen) sog. „Empfangszimmer des Volkes“ sowie bei der Administration des Präsidenten und dem Ministerkabinett sog. „Virtuellen Empfangszimmer“ des Präsidenten bzw. Ministerpräsidenten, die als Art E-Government gelten, eingerichtet. An diesen Empfangszimmern können sich alle Bürger mit ihren Beschwerden gegen Handlungen bzw. Entscheidungen aller Staatsorgane, insbesondere Organe der Exekutive, mit Anfragen oder Problemen wenden. Nach offiziellen Angaben wurden im Jahr 2017 von insgesamt 1454000 Gesuchen von Bürgern 1395000 positiv beschieden. Somit trägt dieses System der Prüfung der Appelle von Bürgern, die mit der Verletzung ihrer Rechte und berechtigten Interessen konfrontiert sind, dazu bei, dass die Bürger in den meisten Fällen nicht vor Gericht gehen, sondern sich direkt an die ersten Personen des Staates wenden. Damit wird jedoch die Illusion erzeugt, die Autorität der höchsten Gewalt, vor allem des Staatsoberhauptes, werde sowohl in der Bevölkerung als auch im gesamten Verwaltungssystem gestärkt. Tatsächlich besteht jedoch die Gefahr, dass sich die Justiz- und Rechtsschutzorgane in einem solchen Rechtsschutzsystem leicht daran gewöhnen, im „Regime manueller Steuerung“ (d.h. entsprechend dem „Befehl von oben“) zu arbeiten. Zu der Tätigkeit der „Empfangszimmer des Volkes" siehe nur: „Wie haben Empfangszimmer des Volkes gearbeitet“. URL: http://www.darakchi.uz/ru/41648 (zuletzt abgerufen am 28.12.2018). Zum Vergleich: während der ersten neun Monate des Jahres 2018 wurden von natürlichen und juristischen Personen 16563 Klagen, die aus öffentlich-rechtlichen Beziehungen resultieren, bei den Verwaltungsgerichten erhoben. Siehe statistischen Daten des Obersten Gerichts der Republik Usbekistan. URL: https://www.oliysud.uz/uploads/2018/11/2018-9-month-adminstrative-courtsstat-uzb.pdf (zuletzt abgerufen am 28.12.2018).

${ }^{68}$ Pudelka, J., Deppe, J. (2017). General administrative law in development in Central Asia. Ost/Mag, pp. 3, 6. URL: https://www.ostinstitut.de/documents/publikationen/Pudelka_Deppe Allgemeines_Verwaltungsrecht_in_Zentralasien_in_der_Entwicklung_OL_2_2017.pdf [Pudelka J., Deppe J. Allgemeines Verwaltungsrecht in Zentralasien in der Entwicklung. 2017. Ost/Mag. URL: https://www.ostinstitut.de/documents/publikationen/Pudelka_Deppe_Allgemeines_ Verwaltungsrecht_in_Zentralasien_in_der_Entwicklung_OL_2_2017.pdf].

${ }^{69}$ Dazu auch Pudelka, J., Deppe, J. (2017). General administrative law in development in Central Asia. Ost/Mag, pp. 32. URL: https://www.ostinstitut.de/documents/publikationen/Pudelka Deppe_Allgemeines_Verwaltungsrecht_in_Zentralasien_in_der_Entwicklung_OL_2_2017.pdf [Pudelk̄a J., Deppe J. Āllgemeines Verwaltungsrecht in Zentralasien in der Entwicklung. $\overline{2}$ 2017. Ost/ Mag. URL: https://www.ostinstitut.de/documents/publikationen/Pudelka_Deppe_Allgemeines Verwaltungsrecht_in_Zentralasien_in_der_Entwicklung_OL_2_2017.pdf]. 
Grundrechte, bei der Anwendung einfachen Gesetzesrechts vor. Es scheint, dass die Hauptgründe dafür im Wesentlichen doktrineller Natur sind. Der Mangel an erforderlichen Theoriekenntnissen an den usbekischen Rechtsfakultäten trägt in besonderem Maße dazu bei, dass die Kluft zwischen dem normativen Geltungsanspruch des Rechts und der Rechtswirklichkeit weiter zunimmt und damit negativ auf das Rechtsbewusstsein der Juristen einwirkt. Im Endeffekt werden so Schwierigkeiten erzeugt, rechtsstaatliche Grundsätze richtig wahrzunehmen und erfolgreich zu implementieren. Diese Faktoren wirken sich nachteilig auf die Juristenausbildung aus und behindern eine nachhaltige Entwicklung des Rechts in Usbekistan.

Der Rechtsstaat braucht rechtspolitisch sensibilisierte Juristen und eine entsprechende Rechtswissenschaft, denn den meisten Juristen gilt die Verpflichtung, den doktrinellen Ansichten einer Rechtsschule zu folgen, als eine Garantie für die Vorhersehbarkeit ihrer Entscheidungen. Es ist auch offensichtlich, dass ein funktionsfähiges und legitimes Rechtssystem dadurch geschaffen wird, dass die Entscheidung der oben genannten Akteure durch die ihnen zur Verfügung stehenden Rechtsquellen und sonstigen Quellen sowie durch allgemein anerkannte Auslegungsmethoden vorgegeben sind.

\section{Schlussbemerkungen}

Das Institut der gerichtlichen Verwaltungskontrolle ist in Usbekistan relativ neu und bisher nur unbefriedigend ausformuliert. Die gerichtliche Verwaltungskontrolle wird auf der Grundlage verschiedener Normen durch die Verwaltungsgerichte, Zivilgerichte, Militärgerichte und Wirtschaftsgerichte ausgeübt.

Verfassungskontrolle wird in Usbekistan auf gesamtstaatlicher Ebene durch das Verfassungsgericht der Republik Usbekistan ausgeübt. Die Verfassungsgerichtsbarkeit hat aber bis jetzt noch keine große praktische Bedeutung erlangt. Es bleibt abzuwarten, ob und wie unter den neuen politischen Bedingungen in Usbekistan die formal-rechtlichen Prüfungskompetenzen des Verfassungsgerichts tatsächlich genutzt werden können, so dass das Verfassungsgericht dennoch einen maßgeblichen, bisher ausgebliebenen Beitrag zur Entwicklung von Verfassungs- und Rechtsstaatlichkeit leisten kann.

Eine Reihe von verwaltungsrechtlichen Streitigkeiten wurde noch in der Sowjetzeit für justitiabel erklärt. Theorie und Praxis der gerichtlichen Verwaltungskontrolle beschritten den Weg einer allmählichen Erweiterung der Prüfungskompetenzen der ordentlichen Gerichte, bis im Jahr 1988 durch das sowjetische Gesetz „Über die Beschwerdeführung bei Gericht gegen rechtswidrige Handlungen von Amtspersonen, die die Rechte der Bürger schmälern“ eine Generalklausel eingeführt wurde, die den Rechtsweg gegen sämtliche Handlungen (Entscheidungen) von Verwaltungsorganen und Amtspersonen zu den ordentlichen Gerichten eröffnete. Dabei wurde das allgemeine Recht auf Beschwerde bei Gericht aus dem allgemeinen Beschwerde- und Antragsrecht (bei den Behörden) entwickelt, das letztlich durch die Verfassung der Republik Usbekistan (Art. 44) garantiert wurde und durch das bis 12.10.2018 geltende Gesetz „Über die Beschwerdeführung bei Gericht gegen Handlungen und Entscheidungen, welche die Rechte und Freiheiten der Bürger verletzen“"vom 30.08.1995 realisiert wurde. Nachdem im Jahre 2017 erstmals das Gerichtssystem Usbekistans um eine spezielle Verwaltungsgerichtsbarkeit ergänzt und im Jahre 2018 das Gesetzbuch der Republik 
Usbekistan über die Verwaltungsrechtspflege und das Gesetz der Republik Usbekistan „Über administrative Prozeduren“ verabschiedet wurden, wurde es offensichtlich, dass das Land den Anschluss an die Entwicklungstendenzen des Verwaltungsverfahrens- und Verwaltungsprozessrechts der kontinentaleuropäischen Staaten sucht.

Natürlich ist die Ausgestaltung des Rechtsinstituts in großem Maße in den gesamten Reformprozess eingebunden und von der gesamten Rechtsentwicklung abhängig. Das Gesetzbuch der Republik Usbekistan über die Verwaltungsrechtspflege weist große Regelungslücken und Mängel auf. So regeln die Bestimmungen des Gesetzbuches das Gerichtsverfahren nur unzureichend und stellen dadurch dem Verwaltungsgericht nicht das nötige Werkzeug für die gerichtliche Prüfung bereit. Zudem erschweren die Inkonsequenz des Gesetzgebers, was die Festlegungen auf einheitliche Terminologie und allgemeine Grundsätze eines rechtsstaatlichen Anforderungen entsprechenden Verwaltungsprozesses anbetrifft, und die Abwesenheit klarer Mechanismen zur Einbringung des öffentlichen Interesses in den Verwaltungsprozess die Anwendung der Normen sowohl durch die Verwaltungsorgane als auch die Gerichte. Dessen ungeachtet stellt das Gesetzbuch „Über die Verwaltungsrechtspflege“ einen weiteren großen Schritt auf dem Weg zu einem demokratischen Rechtstaat dar.

Die Wirtschaftsprozessordnung der Republik Usbekistan enthält einen Katalog verwaltungsrechtlicher Klagearten, regelt jedoch ebenfalls kein spezielles Verfahren für verwaltungsrechtliche Streitigkeiten. Es wäre deshalb richtig und praktischer, den Verwaltungsprozess in einem einheitlichen Gesetzbuch zu regeln und die Verwaltungssachen gänzlich an spezielle Verwaltungsgerichte $\mathrm{zu}$ übertragen. Als vorrangig erscheint dem Autor zunächst jedoch die Vervollkommnung und Ausgestaltung des Instituts der gerichtlichen Verwaltungskontrolle.

Wenn sich der Bürger gegen Verwaltungshandeln zur Wehr setzt, so nutzt er dazu vor allem den traditionellen Weg über die Staatsanwaltschaft oder wendet sich an die Administration des Präsidenten bzw. das Ministerkabinett. Die Gründe für die Unpopularität der Beschwerdeführung bei Gericht liegen zum einen in der Gewöhnung an das Rechtsinstitut der staatsanwaltlichen Aufsicht, wohl aber vor allem in dem bisher nur unbefriedigend ausformulierten Rechtsinstitut der gerichtlichen Verwaltungskontrolle. Zu berücksichtigen sind auch das mangelnde an Demokratie und Rechtsstaat orientierte Rechtsbewusstsein der Bürger und der für die rechtsstaatlichen Reformprozesse relevanten Akteure (Beamten der Exekutive, Richter, Staatsanwälte, Rechtsanwälte und Studenten der Rechtswissenschaft) sowie die Abwesenheit klarer Vorstellungen vom Wesen und von den Besonderheiten des Verwaltungsrechtschutzes im Kontext der rechtsstaatlichen Modernisierung des Staates.

Der erste und verantwortungsvolle Schritt zur Behebung dieser Mängel sollte die Schaffung einer wissenschaftlich fundierten Konzeption des Aufbaus und der Funktionsweise eines modernen Verwaltungsrechtsschutzsystems sein. In diesem Zusammenhang kann die Intensivierung des Austausches zwischen usbekischen und deutschen Rechtswissenschaftlern und Rechtspraktikern eine besondere Rolle spielen, um Gemeinsamkeiten im Rechtsdenken und vor allem beim Problemlösungsbedarf herauszuarbeiten. 